\title{
Binary Choice Models with Discrete Regressors: Identification and Misspecification
}

\author{
Tatiana Komarova * \\ London School of Economics
}

January 8, 2009

\begin{abstract}
In semiparametric binary response models, support conditions on the regressors are required to guarantee point identification of the parameter of interest. For example, one regressor is usually assumed to have continuous support conditional on the other regressors. In some instances, such conditions have precluded the use of these models; in others, practitioners have failed to consider whether the conditions are satisfied in their data. This paper explores the inferential question in these semiparametric models when the continuous support condition is not satisfied and all regressors have discrete support. I suggest a recursive procedure that finds sharp bounds on the parameter of interest and outline several applications. After deriving closed-form bounds on the parameter, I show how these formulas can help analyze cases where one regressor's support becomes increasingly dense. Furthermore, I investigate asymptotic properties of estimators of the identification set. I also propose three approaches to address the problem of empty identification sets when a model is misspecified. Finally, I present a Monte Carlo experiment and an empirical illustration to compare several estimation techniques.
\end{abstract}

JEL Classification: C2, C10, C14, C25

Keywords: Binary response models; Discrete regressors; Partial identification; Misspecification; Linear programming

*London School of Economics and Political Science, Houghton Street, London WC2A 2AE, England. Tel.: +44-(0)20-7852-3707; fax: + 44-(0)20-7955-6592. E-mail: t.komarova@lse.ac.uk. I am deeply indebted to Elie Tamer for many helpful discussions, guidance and encouragement throughout this project. I am very grateful to Joel Horowitz, Charles Manski and Roza Matzkin for their valuable advice and suggestions. I thank the seminar participants at Oxford University. I appreciate comments from Maria Ponomareva, Alexey Tetenov and Ija Trapeznikova. 


\section{Introduction}

The econometrics literature on inference in semiparametric binary response models have used support conditions on observable regressors to guarantee point identification of the vector parameter of interest. These support conditions always require continuity of one (or more) regressors. In practice though, it is not uncommon to have data sets where all regressors have discrete support, such as age, years of education, number of children and gender. In these cases, the parameter of interest is not point identified, that is, a large set of parameters will be consistent with the model. Therefore, it is important to develop methods of drawing accurate inferences without continuous support conditions on the data.

This paper examines the question of identification in semiparametric binary response models in the absence of continuity. Consider

$$
Y=1(X \beta+U \geq 0)
$$

where $Y$ is an observable binary outcome, $U$ is an unobservable, real-valued, scalar random variable, $\beta$ is a $k$-dimensional parameter, and $X$ is an observable random variable with discrete support. I impose a weak median condition on the error term, as in Manski (1985):

$$
M(U \mid X=x)=0 \quad \text { for any } x \text { in support of } X .
$$

In this framework, I provide a methodologically new approach to the analysis of binary response models. The paper makes the following contributions.

I note that the parameter's identification region is described by a system of a finite number of linear inequalities and therefore represents a convex polyhedron. To construct this system, it is enough to know whether conditional probabilities $P(Y=1 \mid X=x)$ are greater or less than 0.5. As was shown by Manski and Thompson (1986), under the median condition the sign of index $x \beta$ is the same as the sign of $P(Y=1 \mid X=x)-0.5$. Moreover, Manski (1988) used this fact to establish a general non-identification result for the case of discrete regressors. The first contribution of the paper is to provide a recursive procedure that allows us to easily find sharp bounds on the identification region. Although this approach was outlined, for example, in Kuhn (1956) and Solodovnikov (1977), it has not been used in the context of identification.

I derive formulas for bounds on parameters, which prove useful in analyzing cases when the support of one regressor can become increasingly dense. Furthermore, I show that the recursive procedure can be used not only to find sharp bounds but also to determine other characteristics of the identification region. Moreover, it can be employed in the extrapolation problem when we want to learn about $P\left(Y=1 \mid X=x_{0}\right)$ for a point $x_{0}$ that is off the support. In addition, because identification regions in ordered response and in single-index models with a monotone link function are described by systems of linear 
inequalities, the recursive procedure can be applied to them too.

Another contribution of the paper is to link binary response models to support vector machines (SVMs) in statistical learning theory. When the support of $X$ is discrete and the median condition $(M)$ holds, binary response models classify points in the support into two groups and every parameter value from the identification set defines a hyperplane that separates these groups. SVMs, in their turn, is a learning technique that focuses on finding a special hyperplane that efficiently separates two classes of given training data. The major difference is that binary response models aim to find all separating hyperplanes, whereas SVMs seek only one hyperplane.

Because models might carry some degree of specification error, the recursive procedure may cease working in some situations. Therefore, it is important to develop techniques that address the consequences of model misspecification. The third contribution of this paper is to offer several methods for dealing with the issue, all of which are based on the optimization of certain objective functions. One approach is the maximum score estimation method presented in Manski $(1975,1985)$. Another allows us to measure the degree of misspecification by finding the minimal number of classification errors. Each method features a crucial property: The set of solutions coincides with the identification set when the model is well specified. The third approach is a modification of a soft margin hyperplane approach in SVMs and it lets us determine the extent of misspecification by determining the minimal size of a general classification error. For a well specified model, this approach gives the closure of the identification set.

Another contribution of this paper is to explore the estimation of the identification region. Although this paper focuses on identification, it is of interest to analyze cases where conditional probabilities $P(Y=1 \mid X=x)$ are not known, but their estimates $\hat{P}(Y=1 \mid X=x)$ are available. In this situation, we can find estimators of identification sets from a system of linear inequalities that uses $\hat{P}(Y=1 \mid X=x)$ instead of $P(Y=1 \mid X=x)$. I show that when the model is well specified, such set estimators converge to the true identification set arbitrarily fast (in terms of Hausdorff distances). I find that the sets of maximum score estimates possess the same property. I also construct confidence regions for the identification set and show that because of the discrete nature of the problem, they are usually conservative.

The last contribution of this paper is an empirical application. The empirical portion of this paper consists of two parts. The first presents the results of a Monte Carlo experiment with a well-specified model. The error term satisfies the median condition but is not independent of the regressors. I show that the estimator of the identification set obtained from the system of inequalities that uses estimated conditional probabilities and the set of maximum score estimates coincides with the identification set. For parameters corresponding to non-constant regressors, I find the set of maximum rank correlation estimates, which turn out to lie inside the identification set but form a much smaller set. 
I also present normalized probit and logit estimates. Though these estimates are located inside the identification set, they are far from the value of the parameter, which was used to generate the model.

The second empirical part is based on data regarding the labor force participation of married women. The decision of women to participate in the labor force is treated as a dependent binary variable and regressed on education, age, labor market experience and number of children. I use different estimation techniques and compare their results. Given that misspecification or sampling error leaves the system of inequalities constructed from the estimates of conditional probabilities without solutions, I use methods suggested for dealing with the misspecification problem. I also find normalized probit and logit estimates, ordinary least squares and least absolute deviation estimates, and compare them to other estimates.

This paper is related to two strands of the literature. The first one embodies a considerable amount of work on partially identified models in econometrics. Studies on partial identification were largely initiated and advanced by Manski (see, for example, Manski (1990, 1995, 2003)), Manski and Tamer (2002) and carried further by other researchers.

The second strand analyzes models with discrete regressors. This topic is relatively underdeveloped in econometric theory, in spite of its importance for empirical work. An example of a recent paper that touches upon this subject is Honore and Tamer (2006). The authors describe how to characterize the identification set for dynamic random effects discrete choice models when points in the support have discrete distributions. For singleindex models $E(Y \mid X=x)=\phi_{\theta}(x \theta)$ with discrete explanatory variables and no assumption on the link function $\phi_{\theta}$ except for measurability, Bierens and Hartog (1988) show that there is an infinite number of observationally equivalent parameters. In particular, the identification set of the $k$-dimensional parameter $\theta=\left(\theta_{1}, \ldots, \theta_{k}\right)$ normalized as $\theta_{1}=1$ will be whole space $\Re^{k-1}$, with the exception of a finite number of hyperplanes (or a countable number of hyperplanes if the regressors have a discrete distribution with an infinite number of values). In binary response models with discrete regressors, Manski (1988) provides a general non-identification result and Horowitz (1998) demonstrates that the parameter can be identified only in very special cases. Magnac and Maurin (2005) also address identification issues in binary response models with discrete regressors. Their framework, however, is different from the framework in this paper. They consider a case where there is a special covariate among the regressors and assume that the model satisfies two conditions related to this covariate - partial independence and large support conditions.

The rest of the paper is organized as follows. Section 2 explains the problem and defines the identification set. Section 3 contains the mathematical apparatus, describes the recursive procedure and its applications and draws an analogy to SVMs. It also outlines applications of the recursive procedure, in particular to single-index and ordered-response models. Section 4 considers misspecification issues and suggests techniques for dealing 
with them. Section 5 analyzes the case in which the discrete support of regressors grows increasingly dense. Section 6 considers the estimation of the identification set from a sample and statistical inference. Section 7 is an empirical section that contains the results of estimations in a Monte Carlo experiment and an MROZ data application. Section 8 concludes and outlines ideas for future research. The proofs of theorems and propositions are collected in the Appendix.

\section{Identification set}

I begin by reviewing the main point identification results in the literature as well as the support conditions that guarantee point identification.

Manski (1985) proved that, when coupled with the median condition $(M)$, the following conditions on the support of $X$ guarantee that $\beta$ in $(B R)$ is identified up to scale:

1. The support of the distribution of $X$ is not contained in any proper linear subspace of $\Re^{k}$.

2. There is at least one component $X_{h}, h \in\{1, \ldots, k\}$ with $\beta_{h} \neq 0$ such that for almost every $\widetilde{x}=\left(x_{1}, \ldots, x_{h-1}, x_{h+1}, \ldots, x_{k}\right)$, the distribution of $X_{h}$ conditional on $\widetilde{X}=\widetilde{x}$ has a density positive almost everywhere with respect to the Lebesgue measure.

In particular, if we normalize $\beta_{1}=1$, then $\beta$ is identified.

The smoothed maximum score method described in Horowitz (1992) and the maximum rank correlation method presented in Han (1987) require these conditions. Klein and Spady's (1993) approach, on the other hand, imposes a stronger assumption: At least one component of $X$ must be a continuous random variable.

It is worth mentioning that Manski (1988) presents other identification results. For instance, under certain conditions, even when $\beta$ is not identified, the signs of $\beta_{1}, \ldots, \beta_{k}$ can be identified. Horowitz (1998) contains a thorough review of identification results for binary response models.

Now I turn to the case of discrete support. Let $X$ be a random variable with the discrete finite support

$$
S(X)=\left\{x^{1}, \ldots, x^{d}\right\}
$$

Following Manski and Thompson (1986), I notice that the median condition allows us to rewrite the binary response model in a form that contains only conditional probabilities $P\left(Y=1 \mid X=x^{l}\right)$ and linear inequalities. Because

$$
\operatorname{Pr}(Y=1 \mid X=x)=\operatorname{Pr}(U \geq-x \beta \mid X=x)=1-\operatorname{Pr}(U<-x \beta \mid X=x),
$$


the median condition implies that

$$
\operatorname{Pr}(Y=1 \mid X=x) \geq 0.5 \quad \Leftrightarrow \quad x \beta \geq 0 .
$$

Thus, model $(B R)$ together with $(M)$ is equivalent to model $(B R M)$, and the identification problem comes down to solving a system of inequalities. Manski and Thompson (1989) interpret condition $(B R M)$ as a "single-crossing" condition for response probabilities. Because $S(X)$ contains a finite number of points, the number of inequalities in the system is also finite. If $\operatorname{Pr}\left(Y=1 \mid X=x^{l}\right) \geq 0.5$, the inequality corresponding to $x^{l}$ is

$$
z_{l 1}+z_{l 2} \beta_{2}+\ldots+z_{l k} \beta_{k} \geq 0
$$

where $z_{l}=x^{l}$. If $\operatorname{Pr}\left(Y=1 \mid X=x^{l}\right)<0.5$, the inequality corresponding to $x^{l}$ is

$$
z_{l 1}+z_{l 2} \beta_{2}+\ldots+z_{l k} \beta_{k}>0
$$

where $z_{l}=-x^{l}$. Though this system contains strict and non-strict inequalities, for the sake of notational convenience, I will write it as encompassing non-strict inequalities

$$
z_{l 1}+z_{l 2} \beta_{2}+\ldots+z_{l k} \beta_{k} \geq 0, \quad l=1, \ldots, d
$$

It is important to keep in mind, however, that some inequalities are strict; this property is what allows us to separate the points with $P(Y=1 \mid X=x) \geq 0.5$ from the points with $P(Y=1 \mid X=x)<0.5$.

Throughout this paper, I will use normalization $\beta_{1}=1$, along with the notations $x^{l}$, which will denote points in the support, and $d$, which will stand for the number of these points, as in (2.1). I will assume that all points in the support are different. Furthermore, I will use $q^{l}$ to signify the probability of $x^{l}$ in the population and $P^{l}$ to indicate conditional probabilities $P\left(Y=1 \mid X=x^{l}\right)$, assuming that $0<q^{l}<1$ for any $l$. The parameter's identification set will be denoted as $B$.

$N$ will stand for the number of observations in a sample, $\hat{q}_{N}^{l}$ will denote the sample frequency estimate of $q^{l}$ and $\hat{P}_{N}^{l}$ will signify the estimate of $P^{l}$. The sample estimate of $B$ will be denoted as $B_{N}$. Throughout the paper, $z_{l}=\operatorname{sgn}\left(P^{l}-0.5\right) x^{l}$, where function $\operatorname{sgn}(\cdot)$ is defined as

$$
\operatorname{sgn}(t)= \begin{cases}1, & t \geq 0 \\ -1, & t<0 .\end{cases}
$$

In several instances $z_{l}$ will mean $z_{l}=\operatorname{sgn}\left(\hat{P}_{N}^{l}-0.5\right) x^{l}$. These cases will be clear from the context.

Theorem 2.1. Parameter $\beta$ in (BRM) is identified up to a $k_{0}$-dimensional convex polyhedron, where $k_{0} \leq k-1$. 
Corollary 2.2. Each $\beta_{m}, m \neq 1$, in $(B R M)$ is identified up to a connected interval.

Theorem 2.1 does not need any proof: It is a direct consequence of the definition of convex polyhedra. To prove Corollary 2.2, I note that the identification interval for $\beta_{m}$, $m \neq 1$, is the projection of the identification set on the axis $x_{l}$. Due to the convexity of $B$, this interval is a connected set.

A few words about convex polyhedra are in order. By definition, a set $B \subset \Re^{k-1}$ is a convex polyhedron if and only if it is the intersection of a finite number of closed half-spaces in $\Re^{k-1}$ space. Formally, a set $B \subset \Re^{k-1}$ is a convex polyhedron if and only if there is an $m \times(k-1)$ matrix $H$ and a vector $h$ of $m$ real numbers such that $B=\left\{b \in \Re^{k-1}: H b \leq h\right\}$.

According to this formal definition, the identification set in $(B R M)$ is not a standard convex polyhedron because the system of linear inequalities defining $B$ contains strict and non-strict inequalities. For our purposes, however, $B$ can be considered a convex polyhedron, keeping in mind that it does not contain some surface points.

The solution set of a finite system of linear inequalities in a finite number of variables is not the only representation of a convex polyhedron. Because every bounded polyhedron has a double description, it can also be described pointwise as the convex hull of a finite number of points. (The minimal set of such points is called the set of the vertices of the polyhedron.) Any unbounded convex polyhedron can be represented as a Minkowski sum of a bounded convex polyhedron and a convex cone (see, for example, Padberg (1999)).

Several authors provide algorithms for finding all the vertices of convex polyhedra when they are described by systems of inequalities. Examples of such works are Motzkin et. al. (1953), Balinski (1961), Chernikova (1965), Manas and Nedoma (1968), Matheiss (1973) and Matheiss and Rubin (1980). Though applicable in practice, these methods have not proved useful in theoretically analyzing the properties of convex polyhedra.

An easier, effective approach in the theoretical analysis of the identification set $B$ in $(B R M)$ is finding the smallest rectangular superset of $B$. This rectangle is the Cartesian product of the identification intervals for $\beta_{m}, m \neq 1$. Its dimension can be smaller than $k-1$ if some $\beta_{m}, m \neq 1$, are point identified.

Figure 1 shows an identification set on the left and its smallest rectangular superset on the right.

\section{Mathematical tools}

\subsection{Recursive method}

In this section, I describe a recursive procedure that finds identification intervals for $\beta_{m}$, $m \neq 1$. Applied to a system of linear inequalities, this method excludes from the system one unknown variable at each step until only a single variable is left. From there, identifying the sharp bounds for the remaining variable is straightforward. Although this approach 

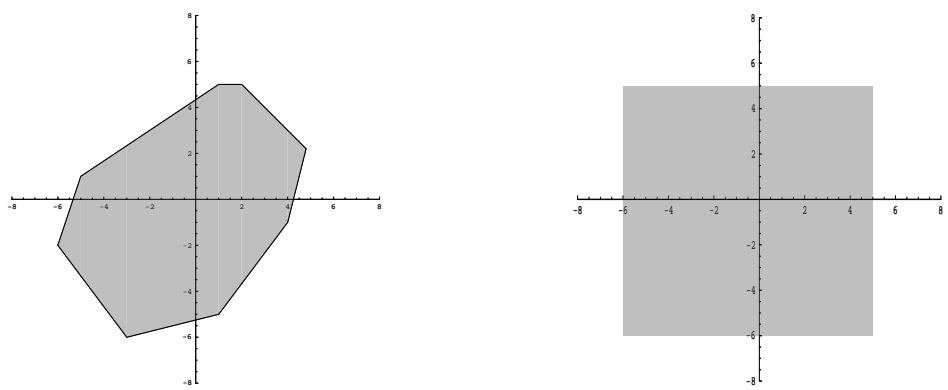

Figure 1. Convex polyhedron and its smallest rectangular superset

is outlined, for instance, in Kuhn (1956) and Solodovnikov (1977), I supplement it by discussing cases in which the system has an unbounded or empty set of solutions and by deriving formulas for parametric bounds.

Consider an arbitrary system of linear inequalities with $k-1$ unknown variables:

$$
\begin{array}{r}
z_{11}+z_{12} b_{2}+\ldots+z_{1 k} b_{k} \geq 0 \\
z_{21}+z_{22} b_{2}+\ldots+z_{2 k} b_{k} \geq 0 \\
\ldots \\
z_{d 1}+z_{d 2} b_{2}+\ldots+z_{d k} b_{k} \geq 0 .
\end{array}
$$

Suppose we want to find sharp bounds for variable $b_{k}$. Consider $i$ 's inequality in the system:

$$
z_{i 1}+z_{i 2} b_{2}+\ldots+z_{i k} b_{k} \geq 0
$$

If $z_{i 2}>0$, then this inequality is equivalent to

$$
-\frac{z_{i 1}}{z_{i 2}}-\frac{z_{i 3}}{z_{i 2}} b_{3} \ldots-\frac{z_{i k}}{z_{i 2}} b_{k} \leq b_{2}
$$

If $z_{j 2}<0$, then it is equivalent to

$$
-\frac{z_{j 1}}{z_{j 2}}-\frac{z_{j 3}}{z_{j 2}} b_{3}-\ldots-\frac{z_{j k}}{z_{j 2}} b_{k} \geq b_{2}
$$

Suppose system $\left(S_{1}\right)$ has $I$ inequalities with $z_{.2}>0, J$ inequalities with $z_{.2}<0$ and $M$ inequalities with $z_{\cdot 2}=0$. In this case, $\left(S_{1}\right)$ can be equivalently written as the system

$$
\begin{gathered}
b_{2} \geq D_{i}, \quad i=1, \ldots, I, \\
N_{j} \geq b_{2}, \quad j=1, \ldots, J, \\
Z_{m} \geq 0, \quad m=1, \ldots, M,
\end{gathered}
$$


where $D_{i}, N_{j}, Z_{m}$ do not contain $b_{2}$ and are linear in $b_{3}, \ldots, b_{k}$. This system implies that

$$
\begin{array}{r}
N_{j} \geq D_{i}, \quad i=1, \ldots, I, \quad j=1, \ldots, J \\
Z_{m} \geq 0, \quad m=1, \ldots, M .
\end{array}
$$

$\left(S_{2}\right)$ is a system of linear inequalities with $k-2$ unknown variables. In other words, system $\left(S_{2}\right)$ consists of $M$ inequalities that do not contain $b_{2}$, and $I \times J$ linear combinations of inequalities $i \in I$ and inequalities $j \in J$ with positive coefficients $-z_{j 2}$ and $z_{i 2}$, respectively. This first step is illustrated in example 9.1 in the Appendix.

The next proposition establishes a relationship between the solutions to $\left(S_{1}\right)$ and $\left(S_{2}\right)$. Proposition 3.1. If $\left(b_{2}^{*}, b_{3}^{*}, \ldots, b_{k}^{*}\right)$ is a solution to $\left(S_{1}\right)$, then $\left(b_{3}^{*}, \ldots, b_{k}^{*}\right)$ is a solution to $\left(S_{2}\right)$. If $\left(b_{3}^{*}, \ldots, b_{k}^{*}\right)$ is a solution to $\left(S_{2}\right)$, then there exists $b_{2}$ such that $\left(b_{2}, b_{3}^{*}, \ldots, b_{k}^{*}\right)$ is a solution to $\left(S_{1}\right)$.

I repeat the process above and exclude a variable from $\left(S_{2}\right)$ (for example, $\left.b_{3}\right)$ to obtain a system $\left(S_{3}\right)$ of linear inequalities with unknown variables $\left(b_{4}, \ldots, b_{k}\right)$. I continue repeating this procedure, removing another variable each time, until I obtain a system with only one unknown variable $b_{k}$ :

$$
\begin{array}{r}
A_{s}+B_{s} b_{k} \geq 0, \quad s=1, \ldots, S, \\
C_{q} \geq 0, \quad q=1, \ldots, Q,
\end{array}
$$

where $B_{s} \neq 0, s=1, \ldots, S$. The statement of Proposition 3.1, applied at each step of the recursive process, implies the following fact.

Proposition 3.2. If $\left(b_{2}^{*}, b_{3}^{*}, \ldots, b_{k}^{*}\right)$ is a solution to $\left(S_{1}\right)$, then $b_{k}^{*}$ is a solution to $\left(S_{k-1}\right)$. If $b_{k}^{*}$ is a solution to $\left(S_{k-1}\right)$, then there exists $\left(b_{2}, \ldots, b_{k-1}\right)$ such that $\left(b_{2}, b_{3}, \ldots, b_{k-1}, b_{k}^{*}\right)$ is a solution to $\left(S_{1}\right)$.

When system $\left(S_{k-1}\right)$ is obtained, I find

$$
\begin{aligned}
& \underline{b}_{k}=\max \left\{-\frac{A_{s}}{B_{s}}: B_{s}>0\right\}, \\
& \bar{b}_{k}=\min \left\{-\frac{A_{s}}{B_{s}}: B_{s}<0\right\} .
\end{aligned}
$$

If $\bar{b}_{k}<\underline{b}_{k}$ or $C_{q}<0$ for some $q$, then system $\left(S_{k-1}\right)$ and, therefore, $\left(S_{1}\right)$ do not have solutions. Otherwise, the set of solutions for $\left(S_{k-1}\right)$ is $\left[\underline{b}_{k}, \bar{b}_{k}\right]$. From Proposition 3.2, I conclude that $\underline{b}_{k}$ and $\bar{b}_{k}$ are sharp bounds for $b_{k}$. This last step is illustrated in example 9.2 in the Appendix.

Evidently, to obtain a system with only variable $b_{k}$ in the last step, I can exclude variables $b_{2}, \ldots, b_{k-1}$ in an order different from the one described here. For instance, I 
could exclude variables in the order $b_{k-1}, b_{k-2}, \ldots, b_{2}$. So, if the set of solutions for $\left(S_{1}\right)$ is nonempty and bounded, variables can be eliminated in any order.

Now I turn to cases where the system either does not have solutions or has an unbounded set of solutions. First, I consider what happens when $\left(S_{1}\right)$ has no solution, focusing particularly on situations in which it is easy to detect that the solution set is empty. In this instance, it is intuitive that the recursive procedure will break at some point. Though I have implicitly assumed until now that $\left(S_{1}\right)$ has solutions, it is possible that systems of inequalities derived from econometric models will not have solutions due to model misspecification. At some step in this situation, we may get an obvious contradiction $C \geq 0$ where $C$ is a negative constant. We may also be able to reach the last step of the procedure, only to discover that $\left(S_{k-1}\right)$ contains clear contradictions, such as $C_{q} \geq 0$ where $C_{q}$ is a negative constant or $\bar{b}_{k}<\underline{b}_{k}$. These situations are illustrated in examples 9.3 and 9.4 in the Appendix.

Second, I consider the case in which $\left(S_{1}\right)$ has an unbounded set of solutions, a condition that can be easy to spot if at one step in the process we notice that all the coefficients corresponding to some variable $b_{i}$ have the same strict sign. Then we can conclude right away that the solution set is unbounded. Nevertheless, we may still be able to continue the procedure and learn more about the solution set if there is a variable with coefficients of both signs. These cases are considered in example 9.4 in the Appendix.

Finally, suppose that at some step in the procedure we notice that, first, no variables in the system have both negative and positive signs, and second, each variable has zero coefficients. Then the system needs further investigation because its solution set may be either unbounded or empty.

Clearly, the recursive procedure can be used to find sharp bounds on any parameter. For example, to find the sharp bounds on parameter $b_{3}$, we can exclude $b_{2}$, then $b_{4}, \ldots, b_{k}$ in the manner described.

Example 3.1. Consider a binary response model

$$
Y=1\left(X_{1}+\beta_{2} X_{2}+\beta_{3} X_{3}+U \geq 0\right)
$$

where $X_{1}$ takes values from $\{-5,-4, \ldots, 4,5\}, X_{2}$ is the constant term $\left(X_{2}=1\right)$, and $X_{3}$ takes values from $\{0,1, \ldots, 7\}$. Thus, the support of $X$ contains 88 points. Conditional probabilities $P^{l}, l=1, \ldots, 88$, are determined by the rule

$$
\begin{array}{ccc}
x_{1}^{l}+1.25-0.5 x_{3}^{l} \geq 0 & \Rightarrow & P^{l} \geq 0.5 \\
x_{1}^{l}+1.25-0.5 x_{3}^{l}<0 & \Rightarrow \quad & P^{l}<0.5 .
\end{array}
$$

Because all $P^{l}$ are known, I can construct a system of linear inequalities that defines the 
identification set $B$ :

$$
P^{l} \geq 0.5 \Leftrightarrow x_{1}^{l}+x_{2}^{l} b_{2}+x_{3}^{l} b_{3} \geq 0, \quad l=1, \ldots, 88
$$

The recursive procedure finds the bounds for $\beta_{2}$ and $\beta_{3}$ : $\beta_{2} \in(1,1.6), \beta_{3} \in(-0.6,-0.42877)$. Figure 2 shows both the identification set and bounds.

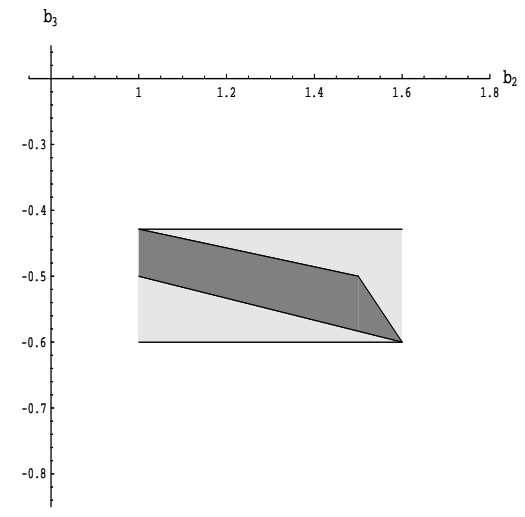

Figure 2. Identification set and its smallest rectangular superset in Example 3.1

\subsection{Formulas for bounds on $\beta_{m}, m \neq 1$}

In this section, I use the recursive procedure to derive formulas for the bounds on $\beta_{m}$, $m \neq 1$, in model $(B R M)$. These bounds are expressed in terms of $z_{i}=\operatorname{sgn}\left(P^{i}-0.5\right) x^{i}$, $i=1, \ldots, d$. For clarity, I first show formulas for the cases $k=3$ and $k=4$ and then present them in general.

Proposition 3.3. Let $k=3$. Suppose the solution set to $\left(S_{1}\right)$ is non-empty and bounded. Then

$$
b_{3}{ }^{l} \leq \beta_{3} \leq b_{3}{ }^{u}, \quad \text { where }
$$

$b_{3}{ }^{u}=\min _{i, j}\left\{-\frac{\left|\begin{array}{cc}z_{j 1} & z_{j 2} \\ z_{i 1} & z_{i 2}\end{array}\right|}{\left|\begin{array}{cc}z_{j 3} & z_{j 2} \\ z_{i 3} & z_{i 2}\end{array}\right|} z_{j 2}<0, z_{i 2}>0,\left|\begin{array}{cc}z_{j 3} & z_{j 2} \\ z_{i 3} & z_{i 2}\end{array}\right|<0\right\}=\min _{i, j}\left\{-\frac{\left|\begin{array}{cc}z_{j 1} & z_{j 2} \\ z_{i 1} & z_{i 2}\end{array}\right|}{\left|\begin{array}{cc}z_{j 3} & z_{j 2}<0 \\ z_{i 3} & z_{i 2}>0\end{array}\right|<0}\right\}$,

$b_{3}^{l}=\max _{i, j}\left\{-\frac{\left|\begin{array}{cc}z_{j 1} & z_{j 2} \\ z_{i 1} & z_{i 2}\end{array}\right|}{\left|\begin{array}{cc}z_{j 3} & z_{j 2} \\ z_{i 3} & z_{i 2}\end{array}\right|} z_{j 2}<0, z_{i 2}>0,\left|\begin{array}{cc}z_{j 3} & z_{j 2} \\ z_{i 3} & z_{i 2}\end{array}\right|>0\right\}=\max _{i, j}\left\{-\frac{\left|\begin{array}{cc}z_{j 1} & z_{j 2} \\ z_{i 1} & z_{i 2}\end{array}\right|}{\left|\begin{array}{cc}z_{j 3} & z_{j 2}<0 \\ z_{i 3} & z_{i 2}>0\end{array}\right|>0}\right\}$. 
Assume there are sets of indices $i, j$ such that the conditions in the definition of $b_{3}{ }^{u}$ and $b_{3}{ }^{l}$ are satisfied. Bounds $b_{3}{ }^{u}$ and $b_{3}{ }^{l}$ are not necessarily sharp.

Let me explain the last statement in this proposition. According to the recursive procedure, if in some inequalities the coefficients corresponding to $b_{2}$ are 0 , then we carry over those inequalities to the next step without any changes. The formulas for $b_{3}{ }^{u}$ and $b_{3}{ }^{l}$, however, ignore situations in which some coefficients corresponding to $b_{2}$ can be 0 . Therefore, they do not necessarily describe sharp bounds. Sharp bounds $\tilde{b}_{3}^{u}$ and $\tilde{b}_{3}^{l}$ that account for these cases can be written as follows:

$$
\tilde{b}_{3}^{u}=\min \left\{b_{3}^{u}, \min _{i}\left\{-\frac{z_{i 1}}{z_{i 3}}: z_{i 2}=0, z_{i 3}<0\right\}\right\}, \tilde{b}_{3}^{l}=\min \left\{b_{3}^{l}, \min _{i}\left\{-\frac{z_{i 1}}{z_{i 3}}: z_{i 2}=0, z_{i 3}>0\right\}\right\} .
$$

If there is a constant term among regressors, these formulas are simpler. Without a loss of generality, $x_{i 2}=1, i=1, \ldots, d$. Then $z_{j 2}<0$ is possible if and only if $z_{j 2}=-1$, and $z_{i 2}>0$ is possible if and only if $z_{i 2}=1$. The formulas can be written as follows:

$$
b_{3}{ }^{u}=\min _{i, j}\left\{-\frac{x_{i 1}-x_{j 1}}{x_{i 3}-x_{j 3}}: x_{i 3}-x_{j 3}<0\right\}, \quad b_{3}{ }^{l}=\max _{i, j}\left\{-\frac{x_{i 1}-x_{j 1}}{x_{i 3}-x_{j 3}}: x_{i 3}-x_{j 3}>0\right\} .
$$

Now I consider the case $k=4$.

Proposition 3.4. Let $k=4$. Suppose the solution set to $\left(S_{1}\right)$ is non-empty and bounded. Then

$$
\begin{aligned}
& b_{4}{ }^{l} \leq \beta_{4} \leq b_{4}{ }^{u} \text {, where }
\end{aligned}
$$

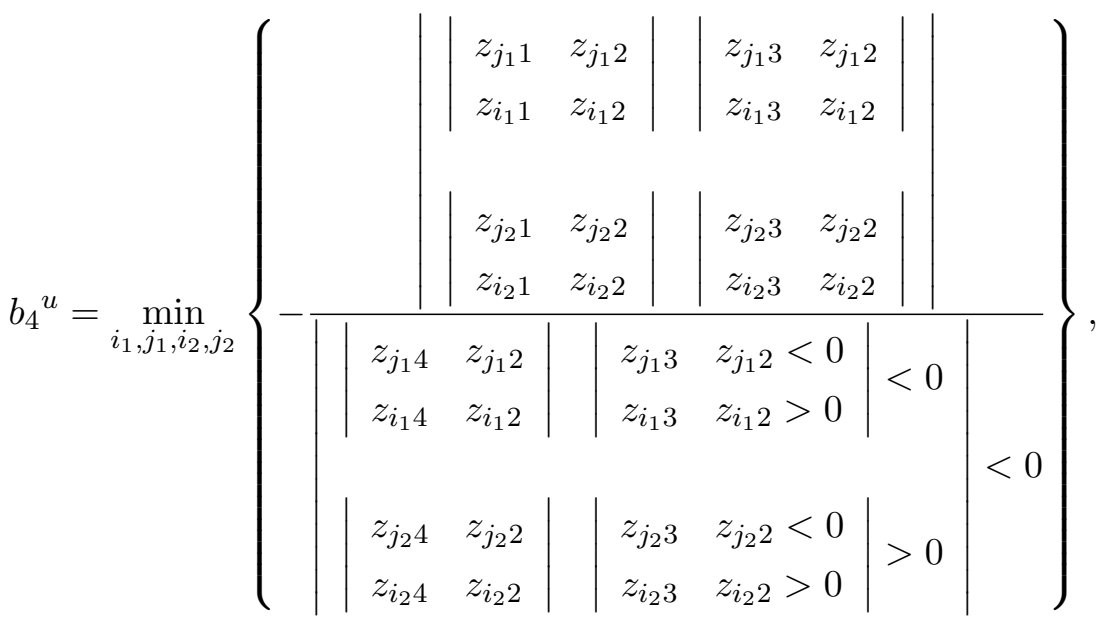




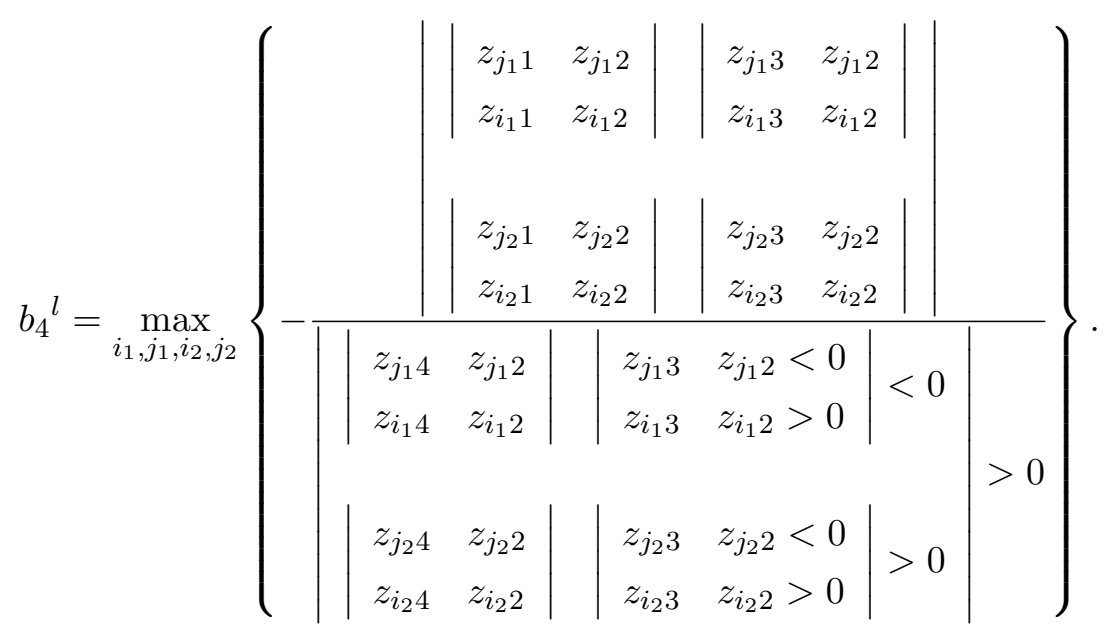

Assume there are sets of indices $i_{1}, i_{2}, j_{1}, j_{2}$ such that the conditions in the definition of $b_{4}{ }^{u}$ and $b_{4}{ }^{l}$ are satisfied. Bounds $b_{4}{ }^{u}$ and $b_{4}{ }^{l}$ are not necessarily sharp.

The reason why $b_{4}{ }^{u}$ and $b_{4}{ }^{l}$ are not necessarily sharp is similar to the explanation described earlier, when I considered the case of $k=3$ : The formulas ignore instances in which some inequalities are carried over to the next step without any changes. When $k>3$, it becomes difficult to keep track of these inequalities at each step of the procedure. such inequalities; that is why I do not give formulas for sharp bounds for $k>3$.

It is worth noticing the symmetry of the formulas for $b_{3}^{l}$ and $b_{3}^{u}$, as well as for $b_{4}^{l}$ and $b_{4}^{u}$. To find the lower (upper) bounds $b_{3}^{l}$ or $b_{4}^{l}\left(b_{3}^{u}\right.$ or $\left.b_{4}^{u}\right)$, we choose inequalities for which the determinant in the denominator is positive (negative). This symmetry will allow me to formulate results in section 5, when I consider the situation in which the support of $X$ becomes increasingly dense.

Proposition 3.4 is a special case of Proposition 3.5, which I formulate below. First, however, let me introduce some notations. Define

$$
A_{1}(m, i, j)=\left|\begin{array}{cc}
z_{j m} & z_{j 2} \\
z_{i m} & z_{i 2}
\end{array}\right|
$$

where $z_{j 2}, z_{i 2}$ are such that $z_{j 2}<0, z_{i 2}>0$. Let me write this formula as

$$
A_{1}(m, i, j)=\left|\begin{array}{cc}
z_{j m} & z_{j 2}<0 \\
z_{i m} & z_{i 2}>0
\end{array}\right|
$$

Then, according to Proposition 3.3,

$$
b_{3}{ }^{u}=\min _{i, j}\left\{-\frac{A_{1}(1, i, j)}{A_{1}(3, i, j)<0}\right\}, \quad b_{3}{ }^{l}=\max _{i, j}\left\{-\frac{A_{1}(1, i, j)}{A_{1}(3, i, j)>0}\right\} .
$$


Note that $A_{1}(m, i, j)$ can be written in the form

$$
A_{1}\left(m, i_{1}, j_{1}, i_{2}, j_{2}\right)=\left|\begin{array}{ll}
A_{0}(m, j) & A_{0}(2, j)<0 \\
A_{0}(m, i) & A_{0}(2, i)>0
\end{array}\right|,
$$

where $A_{0}(m, i)=z_{i m}$. Let

$$
A_{2}\left(m, i_{1}, j_{1}, i_{2}, j_{2}\right)=\left|\begin{array}{cc}
A_{1}\left(m, i_{1}, j_{1}\right) & A_{1}\left(3, i_{1}, j_{1}\right)<0 \\
A_{1}\left(m, i_{2}, j_{2}\right) & A_{1}\left(3, i_{2}, j_{2}\right)>0
\end{array}\right|
$$

Then $b_{4}{ }^{u}, b_{4}{ }^{l}$ from Proposition 3.4 can be written as

$$
b_{4}{ }^{u}=\min _{i_{1}, j_{1}, i_{2}, j_{2}}\left\{-\frac{A_{2}\left(1, i_{1}, j_{1}, i_{2}, j_{2}\right)}{A_{2}\left(4, i_{1}, j_{1}, i_{2}, j_{2}\right)<0}\right\}, \quad b_{4}{ }^{l}=\max _{i_{1}, j_{1}, i_{2}, j_{2}}\left\{-\frac{A_{2}\left(1, i_{1}, j_{1}, i_{2}, j_{2}\right)}{A_{2}\left(4, i_{1}, j_{1}, i_{2}, j_{2}\right)>0}\right\} .
$$

Now I can formulate a general result.

Proposition 3.5. Let $k \geq 3$. Suppose the solution set to $\left(S_{1}\right)$ is non-empty and bounded. Then

$$
b_{k}^{l} \leq \beta_{k} \leq b_{k}^{u}
$$

where

$$
\begin{aligned}
& b_{k}{ }^{u}=\min _{i_{1}, \ldots, j_{2 k-3}}\left\{-\frac{A_{k-2}\left(1, i_{1}, \ldots, j_{2^{k-3}}\right)}{A_{k-2}\left(k, i_{1}, \ldots, j_{2^{k-3}}\right)<0}\right\}, \\
& b_{k}^{l}=\max _{i_{1}, \ldots, j_{2^{k-3}}}\left\{-\frac{A_{k-2}\left(1, i_{1}, \ldots, j_{2^{k-3}}\right)}{A_{k-2}\left(k, i_{1}, \ldots, j_{2^{k-3}}\right)>0}\right\},
\end{aligned}
$$

where $A_{k-2}\left(m, i_{1}, \ldots, j_{2^{k-3}}\right)$ is defined recursively as

$$
\begin{aligned}
& A_{k-2}\left(m, i_{1}, \ldots, j_{2^{k-3}}\right)=\left|\begin{array}{cl}
A_{k-3}\left(m, i_{1}, \ldots, j_{2^{k-4}}\right) & A_{k-3}\left(k-1, i_{1}, \ldots, j_{2^{k-4}}\right)<0 \\
A_{k-3}\left(m, i_{2^{k-4}+1}, \ldots, j_{2^{k-3}}\right) & A_{k-3}\left(k-1, i_{2^{k-4}+1}, \ldots, j_{2^{k-3}}\right)>0
\end{array}\right|, \\
& A_{0}(m, i)=z_{i m} .
\end{aligned}
$$

Assume there are sets of indices $i_{1}, \ldots, i_{2^{k-3}}, j_{1}, \ldots, j_{2^{k-3}}$ such that the conditions in the definition of $b_{k}{ }^{u}$ and $b_{k}{ }^{l}$ are satisfied. Bounds $b_{k}{ }^{u}$ and $b_{k}{ }^{l}$ are not necessarily sharp.

\subsection{Applications of the recursive method}

The recursive method's effectiveness extends well beyond identification intervals. It can come in handy, for instance, when we are interested in some specific properties of $B$ or other related questions. This section presents several applications of the method. 


\subsubsection{A rectangular subset with the largest perimeter}

Because not every point in the smallest rectangular superset of $B$ belongs to $B$, it may be of interest to find subsets of $B$ that have certain properties. The recursive method allows us to easily find a rectangular subset of $B$ with the largest possible perimeter. For simplicity, consider the case where $k=3$. The problem that has to be solved is

$$
a_{2}+a_{3} \rightarrow \max _{b_{2}, b_{3}, a_{2}, a_{3}}
$$

subject to

$$
\begin{aligned}
z_{i 1}+z_{i 2} b_{2}+z_{i 3} b_{3} & \geq 0 \\
z_{i 1}+z_{i 2}\left(b_{2}+a_{2}\right)+z_{i 3} b_{3} & \geq 0 \\
z_{i 1}+z_{i 2} b_{2}+z_{i 3}\left(b_{3}+a_{3}\right) & \geq 0 \\
z_{i 1}+z_{i 2}\left(b_{2}+a_{2}\right)+z_{i 3}\left(b_{3}+a_{3}\right) & \geq 0 \\
i=1, \ldots, d & \\
a_{2} \geq 0, \quad a_{3} & \geq 0 .
\end{aligned}
$$

Denote $a=a_{2}+a_{3}$. Substitute expression $a_{3}=a-a_{2}$ into the system, and obtain

$$
\begin{aligned}
z_{i 1}+z_{i 2} b_{2}+z_{i 3} b_{3} & \geq 0 \\
z_{i 1}+z_{i 2} b_{2}+z_{i 3} b_{3}+z_{i 2} a_{2} & \geq 0 \\
z_{i 1}+z_{i 2} b_{2}+z_{i 3} b_{3}-z_{i 3} a_{2}+z_{i 3} a & \geq 0 \\
z_{i 1}+z_{i 2} b_{2}+z_{i 3} b_{3}+\left(z_{i 2}-z_{i 3}\right) a_{2}+z_{i 3} & \geq 0 \\
i=1, \ldots, d & \\
a_{2} \geq 0, \quad a & \geq 0 .
\end{aligned}
$$

Find the upper bound on $a$ from system (3.1), and denote it as $a^{u}$. Let $\left(b_{2}^{*}, b_{3}^{*}, a_{2}^{*}\right)$ be any solution of (3.1) when $a$ is fixed at its highest value: $a=a^{u}$. Value $2 a^{u}$ is the largest possible perimeter, and points $\left(b_{2}^{*}, b_{3}^{*}\right),\left(b_{2}^{*}+a_{2}^{*}, b_{3}^{*}\right),\left(b_{2}^{*}, b_{3}^{*}+a^{u}-a_{2}^{*}\right)$ and $\left(b_{2}^{*}+a_{2}, b_{3}^{*}+a^{u}-a_{2}^{*}\right)$ describe a rectangular subset with this perimeter.

\subsubsection{A cubic subset with the largest volume}

The recursive procedure can also determine a cubic subset of $B$ with the largest volume. Again for simplicity assume $k=3$. In this instance, we look for a square with the largest 
area. Denote the side of a square as $a$ and find the upper bound $a^{u}$ on $a$ in the system

$$
\begin{aligned}
& z_{i 1}+z_{i 2} b_{2}+z_{i 3} b_{3} \geq 0 \\
& z_{i 1}+z_{i 2} b_{2}+z_{i 3} b_{3}+z_{i 2} a \geq 0 \\
& z_{i 1}+z_{i 2} b_{2}+z_{i 3} b_{3}+z_{i 3} a \geq 0 \\
& z_{i 1}+z_{i 2} b_{2}+z_{i 3} b_{3}+\left(z_{i 2}+z_{i 3}\right) a \geq 0 \\
& i=1, \ldots, d \\
& a \\
& a 0
\end{aligned}
$$

Let $\left(b_{2}^{*}, b_{3}^{*}\right)$ be any solution of $(3.2)$ when $a$ is fixed at its largest value: $a=a^{u}$. Value $\left(a^{u}\right)^{2}$ is the largest possible area of a square subset, and points $\left(b_{2}^{*}, b_{3}^{*}\right),\left(b_{2}^{*}+a^{u}, b_{3}^{*}\right),\left(b_{2}^{*}, b_{3}^{*}+a^{u}\right)$ and $\left(b_{2}^{*}+a^{u}, b_{3}^{*}+a^{u}\right)$ describe a square subset with this area.

\subsubsection{Extrapolation}

Suppose we have a point $x^{*}=\left(x_{1}^{*}, \ldots, x_{k}^{*}\right) \notin S(X)$ and would like to learn the value of $P\left(Y=1 \mid X=x^{*}\right)$. Clearly, this value is not identified, but we may be able to determine the sign of $P\left(Y=1 \mid X=x^{*}\right)-0.5$.

We proceed by finding the sharp bounds on the values of linear function

$$
x_{1}^{*}+x_{2}^{*} b_{2}+\ldots+x_{k}^{*} b_{k}, \quad\left(b_{2}, \ldots, b_{k}\right) \in B .
$$

If the lower bound is non-negative, then $P\left(Y=1 \mid X=x^{*}\right)-0.5 \geq 0$. If the upper bound is negative, then $P\left(Y=1 \mid X=x^{*}\right)-0.5<0$. Finally, if the interval between the lower and upper bound contains zero, then we cannot draw any conclusions about the value of $\operatorname{sgn}\left(P\left(Y=1 \mid X=x^{*}\right)-0.5\right)$.

Let me show how we can find these bounds. Introduce a new variable $b^{\prime}$ which is the value of the linear function:

$$
b^{\prime}=x_{1}^{*}+x_{2}^{*} b_{2}+\ldots+x_{k}^{*} b_{k} .
$$

Assume that at least one of $x_{i}^{*}, i=2, \ldots, k$, is different from 0 . Without a loss of generality, $x_{k}^{*} \neq 0$. Then $b_{k}$ can be expressed through $b_{2}, \ldots, b_{k}, b^{\prime}$ as follows:

$$
b_{k}=\frac{1}{x_{k}^{*}} b^{\prime}-\frac{x_{1}^{*}}{x_{k}^{*}}-\frac{x_{2}^{*}}{x_{k}^{*}} b_{2}-\ldots-\frac{x_{k-1}^{*}}{x_{k}^{*}} b_{k-1} .
$$

Substitute this expression into the system that defines the identification set:

$$
z_{l 1}+z_{l 2} b_{2}+\ldots+z_{l k} b_{k} \geq 0, \quad l=1, \ldots, d
$$


Then obtain a system of linear inequalities with unknown variables $b_{2}, \ldots, b_{k-1}, b^{\prime}$ :

$$
z_{l 1}-z_{l k} \frac{x_{1}^{*}}{x_{k}^{*}}+\left(z_{l 2}-z_{l k} \frac{x_{2}^{*}}{x_{k}^{*}}\right) b_{2}+\ldots+\left(z_{l, k-1}-z_{l k} \frac{x_{k-1}^{*}}{x_{k}^{*}}\right) b_{k-1}+\frac{z_{l k}}{x_{k}^{*}} b^{\prime} \geq 0, \quad l=1, \ldots, d .
$$

Using the recursive procedure, we can find the sharp bounds for $b^{\prime}$ - that is, the sharp bounds on the values of the linear function.

\subsubsection{Single-index models}

Consider a single-index model

$$
E(Y \mid X=x)=G(x \beta)
$$

with an increasing function $G$. Normalize $\beta_{1}=1$. The identification set is the set of $\left(b_{2}, \ldots, b_{k}\right)$ such that $b=\left(1, b_{2}, \ldots, b_{k}\right)$ solve the following system of linear inequalities:

$$
\forall\left(x^{l}, x^{m} \in S(X)\right) \quad E\left(Y \mid X=x^{l}\right)>E\left(Y \mid X=x^{m}\right) \Rightarrow x^{l} b>x^{m} b .
$$

If, for example, $E\left(Y \mid X=x^{1}\right)>E\left(Y \mid X=x^{2}\right)>\ldots>E\left(Y \mid X=x^{d}\right)$, then the identification set is described by a system of $d-1$ inequalities:

$$
x^{1} b>x^{2} b>\ldots>x^{d} b .
$$

In general, there may be many more inequalities defining the identification set. For example, if $d$ is even and $E\left(Y \mid X=x^{l}\right)=1$ for $l=1, \ldots, d / 2$, and $E\left(Y \mid X=x^{l}\right)=0$ for $l=d / 2+1, \ldots, d$, then the system describing the identification set comprises $d^{2} / 4$ inequalities:

$$
x^{1} b>x^{m} b, \quad l=1, \ldots, d / 2, \quad m=d / 2+1, \ldots, d .
$$

Applied to the system of inequalities defining the identification set, the recursive procedure finds the sharp bounds on the components of $\beta$.

\subsection{Ordered response models}

In ordered response models, agents choose among $J$ alternatives according to the rule

$$
Y=\sum_{j=1}^{J+1} j 1\left(\alpha_{j-1}<Y^{*} \leq \alpha_{j}\right)
$$

where $\alpha_{0}<\alpha_{1}<\ldots<\alpha_{J}<\alpha_{J+1}, \alpha_{0}=-\infty, \alpha_{J+1}=+\infty, Y^{*}=x \beta+U$. In general, threshold levels $\alpha_{j}$ are not known. If we assume the median condition $M\left(U \mid X=x^{l}\right)=0$, 
$l=1, \ldots, d$, then

$$
P\left(Y \leq j \mid X=x^{l}\right)=P\left(x^{l} \beta+U \leq \alpha_{j}\right)=F_{U \mid x^{l}}\left(\alpha_{j}-x^{l} \beta\right), \quad l=1, \ldots, d, \quad j=1, \ldots, J .
$$

The identification set is described by a system of linear inequalities with a maximum of $2 d+J-1$ inequalities. Each $x^{l}$ contributes one or two inequalities to the system, and each $x^{l}$ has three possibilities: $P\left(Y \leq 1 \mid X=x^{l}\right) \geq 0.5$, or $P\left(Y \leq J \mid x=x^{l}\right)<0.5$, or $P\left(Y \leq 1 \mid X=x^{l}\right)<0.5$ and $P\left(Y \leq J \mid x=x^{l}\right) \geq 0.5$. In the first case, $x^{l}$ contributes the inequality

$$
\alpha_{1}-x^{l} b \geq 0
$$

In the second case, it provides

$$
\alpha_{J}-x^{l} b<0
$$

In the third case, find $j\left(x^{l}\right) \in\{2, \ldots, J\}$ such that

$$
P\left(Y \leq j\left(x^{l}\right)-1 \mid X=x^{l}\right)<0.5, \quad P\left(Y \leq j\left(x^{l}\right) \mid x=x^{l}\right) \geq 0.5
$$

Then $x^{l}$ contributes two inequalities

$$
\alpha_{j\left(x^{l}\right)-1}-x^{l} b<0, \quad \alpha_{j\left(x^{l}\right)}-x^{l} b \geq 0 .
$$

Thus, points from $S(X)$ form a system of at most $2 d$ linear inequalities. However, we also have to add $J-1$ inequalities

$$
\alpha_{j}-\alpha_{j-1}>0, \quad j=2, \ldots, J
$$

Some of these additional inequalities will be excessive because, for example, (3.4) implies that $\alpha_{j\left(x^{l}\right)-1}<\alpha_{j\left(x^{l}\right)}$.

After we normalize $b$ by setting $b_{1}=1$, we get a system of at most $2 d+J-1$ linear inequalities with $k-1+J$ unknown variables $b_{2}, \ldots, b_{k}, \alpha_{1}, \ldots, \alpha_{J}$. This system contains both strict and non-strict inequalities. We can apply the recursive method to find the sharp bounds on the values of these variables.

\subsection{Support vector machines}

In this section, I show a connection between the identification problem in binary response models and support vector machines in statistical learning theory.

To describe this relationship I have to assume that one of the regressors in $X$ is a constant term. Without a restriction of generality, $X_{k}=1$. Define a set $\tilde{S}(X) \subset \Re^{k-1}$ that consists of points from $S(X)$ with an omitted last regressor $x_{k}$. The problem of finding the identification region in $(B R M)$ is the same as determining all the hyperplanes that 

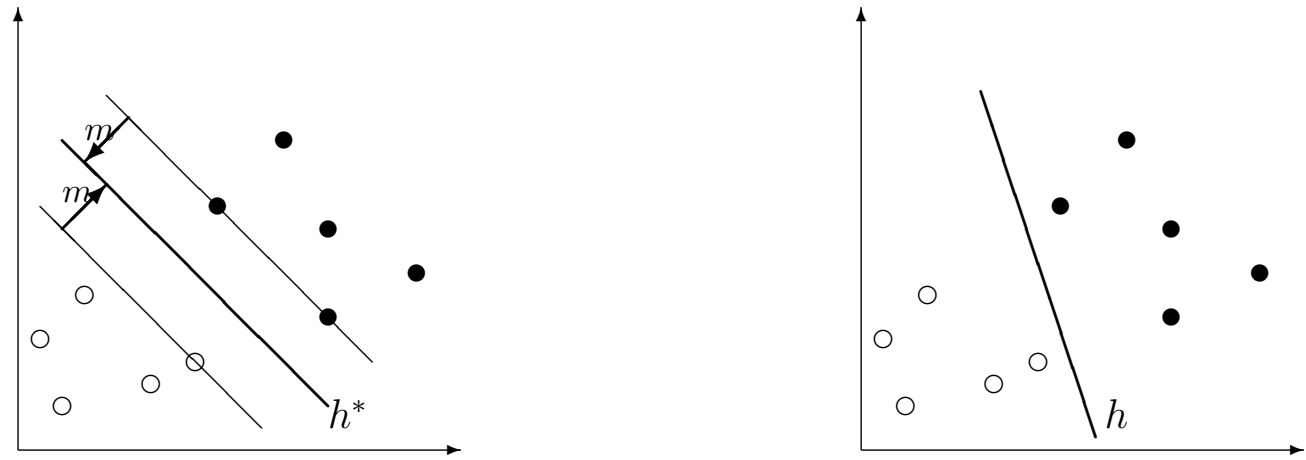

Figure 3. The optimal hyperplane $h^{*}$ with maximal margin $m$ (left) and a non-optimal separating hyperplane $h$ (right)

separate two classes of points from $\tilde{S}(X)$ : those for which $P(Y=1 \mid X=x) \geq 0.5$ and those for which $P(Y=1 \mid X=x)<0.5$.

Support vector machines (SVMs) are learning techniques used for classification problems. They separate a given set of binary-labeled training data with a hyperplane that maximizes the distance (or, the margin) between itself and the closest training data point. It is called the optimal or the maximal margin hyperplane.

SVMs are similar to the identification problem for $(B R M)$ in that both are hyperplanebased classifiers. However, in contrast with SVMs, which select only one hyperplane, the identification set in $(B R M)$ contains all the vectors that define separating hyperplanes. Figure 3 shows optimal and a non-optimal separating hyperplanes for the two classes of points.

Let me briefly present the mathematical description of SVMs (for more details, see Vapnik (2000)). For a better comparison with existing literature, I adopt their notations.

Suppose we have a training set of $n$ independent and identically distributed observations of $(X, Y)$ drawn according to the unknown probability distribution $P(x, y)$ :

$$
\left\{\left(x_{i}, y_{i}\right)\right\}_{i=1}^{n}, x \in \Re^{k}, y \in\{-1,1\},
$$

where observation $i$ is assigned to one of two classes (-1 or 1 ) according to its value $y_{i}$. Also suppose that the two classes of points in the training set can be separated by the linear hyperplane $w_{1} x_{1}+\ldots+w_{k} x_{k}-a=0$. To find the optimal hyperplane, solve the quadratic programming problem

$$
\underset{w_{1}, \ldots, w_{k}, a}{\min } \sum_{j=1}^{k} w_{j}^{2}
$$

subject to

$$
y_{i}\left[w_{1} x_{i, 1}+\ldots+w_{k} x_{i, k}-a\right] \geq 1, \quad i=1, \ldots, n .
$$


The solution of this problem is unique, and it defines the optimal hyperplane.

Let me now return to the identification set $B$ in $(B R M)$. As mentioned earlier, any point in $B$ can serve as a classification rule. Generally, the quality of a classification rule is measured by a loss function. In Lin $(2000,2002)$, the following expected misclassification rate (or generalization error) is regarded as a loss function:

$$
R(\eta)=P(\eta(X) \neq Y)=\int 0.5|y-\eta(x)| d P
$$

where $\eta$ is a classification rule. The classification rule with the smallest generalization error is

$$
\eta^{*}(x)=\operatorname{sgn}(P(Y=1 \mid X=x)-0.5) .
$$

Thus, the median condition $(M)$ in the binary response model $(B R)$ guarantees that any point in the identification set $B$ constitutes a linear decision rule that is optimal with regard to the expected misclassification rate criterion (3.7).

It is important to stress that in this section I assumed that $(B R M)$ is well specified.

\section{Misspecification}

If $(B R M)$ is misspecified, then it is possible that $\left(S_{1}\right)$ has no solutions. One way to handle this situation is to consider an optimization problem with respect to $b$ and find its solution set. This problem has to satisfy two requirements: first, it should be meaningful, allowing us to interpret its set of solutions in terms of the properties of model $(B R)$. Second, its solution set should coincide with the identification set when $(B R M)$ is well specified.

I suggest several optimization techniques to apply when a model is misspecified and discuss their sets of solutions. There is no best method among them, and preferences for a certain approach usually change depending on the situation. Of course, procedures described below are just a few of the possible options. It is also worth mentioning that one of these techniques yields the closure of the identification set, not the identification set itself. However, it can be used to deal with the misspecification problem.

\subsection{Maximum score method}

Manski (1985) defines a maximum score estimator (on the population level) as an estimator that minimizes the expected absolute distance between the conditional expectation of $Y$ conditional on $X$ and $1(X b \geq 0)$ (if the median condition $(M)$ holds, then $M(Y \mid X)=$ $1(X \beta \geq 0))$ :

$$
\min _{b: b_{1}=1} E|E(Y \mid X)-1(X b \geq 0)|
$$


Equivalently, a maximum score estimator minimizes the expected squared distance between between $E(Y \mid X)$ and $1(X b \geq 0)$ :

$$
\min _{b: b_{1}=1} E(E(Y \mid X)-1(X b \geq 0))^{2}
$$

It also solves optimization problems

$$
\begin{gathered}
\min _{b: b_{1}=1} E|Y-1(X b \geq 0)|, \\
\min _{b: b_{1}=1} E(Y-1(X b \geq 0))^{2} .
\end{gathered}
$$

First, let me show that when $(B R M)$ is well specified, the set of maximum score estimates coincides with the identification set. When all the regressors are discrete, optimization problem (4.1) is tantamount to the maximization of the function

$$
S^{m s}(b)=2 \sum_{l=1}^{d} q^{l}\left(P^{l}-0.5\right) \operatorname{sgn}\left(x^{l} b\right)
$$

where, I remind, $q^{l}=P\left(X=x^{l}\right), P^{l}=P\left(Y=1 \mid X=x^{l}\right)$. Let $B^{m s}$ stand for the set of the maximum score estimates:

$$
B^{m s}=\left\{\left(b_{2}, \ldots, b_{k}\right):\left(1, b_{2}, \ldots, b_{k}\right) \in \operatorname{Argmax}_{b: b_{1}=1} S^{m s}(b)\right\}
$$

The next proposition compares sets $B^{m s}$ and $B$.

Proposition 4.1. Suppose that model (BRM) is well specified. Then $B^{\text {ms }}$ is a convex polyhedron and

$$
\begin{array}{ll}
\forall\left(x^{l} \in S(X)\right) P^{l} \neq 0.5 \quad & \Rightarrow \quad B=B^{m s} \\
\exists\left(x^{l} \in S(X)\right) P^{l}=0.5 & \Rightarrow \quad B \subset B^{m s}
\end{array}
$$

with a strict inclusion.

If $(B R M)$ is misspecified, then $B^{m s}$ may be not a convex polyhedron. This may happen, for instance, when many identical values exist among $\left(P^{l}-0.5\right) q^{l}, l=1, \ldots, d$. However, $B^{m s}$ will always be a finite union of several disjoint convex polyhedra.

\subsection{Minimal number of classification errors}

In $(B R M)$ all points from $S(X)$ can be grouped into two classes. If $P^{l} \geq 0.5$, then $x^{l}$ is assigned to that class; otherwise, it is assigned to the other class. If the model is misspecified and $B=\emptyset$, then some classification errors have been made. In order to find the values of $b$ that minimize the number of classification errors, consider the following 
optimization problem:

$$
\max _{b: b_{1}=1} Q(b), \quad \text { where } \quad Q(b)=\sum_{l=1}^{d} \operatorname{sgn}\left(P^{l}-0.5\right) \operatorname{sgn}\left(x^{l} b\right) .
$$

If $(B R M)$ is well specified, the set of solutions for (4.3) coincides with $B$. Because the value of $Q(b)$ is $d$ minus the number of classification errors, the maximization of $Q(b)$ minimizes the number of these errors. I can modify problem (4.3) and consider

$$
Q(b)=\sum_{l=1}^{d} \operatorname{sgn}\left(P^{l}-0.5\right) \operatorname{sgn}\left(x^{l} b\right) q^{l} .
$$

Then the maximization problem is equivalent to the minimization of the expected absolute distance between $M(Y \mid X)$ and $1(X b \geq 0)$ :

$$
\min _{b: b_{1}=1} E|M(Y \mid X)-1(X b \geq 0)|
$$

The solution sets for (4.3) and (4.4) are finite unions of disjoint convex polyhedra.

\subsection{Minimal general classification error}

In this section, I discuss a geometric approach to misspecification. It is based on the soft margin hyperplane method in support vector machines, a technique suggested in Cortes and Vapnik (1995) for handling cases in which two classes of training data are not linearly separable. Roughly speaking, this method deals with errors in the data by allowing some anomalous points to fall on the wrong side of the hyperplane. I describe this approach in more detail in the end of the section.

The method in SVMs chooses only one soft margin hyperplane: the optimal one. We are interested in finding a set of these hyperplanes, however, rather than just one. In fact, we would like to determine the set of all soft margin hyperplanes, if possible. An attractive feature of the approach outlined here is that it only requires solving linear programming problems, so it is easy to implement.

As in section 3.5, first assume that there is a constant term among regressors. Without a restriction of generality, $X_{k}=1$. Let set $\tilde{S}(X) \subset \Re^{k-1}$ consist of points from $S(X)$ with an omitted last regressor $x_{k}$. For now, let me abandon normalization $b_{1}=1$. Without this normalization, the identification set is a subset of $\Re^{k}$. Denote it as $\tilde{B}$. Geometrically, $\tilde{B}$ consists of $k$-dimensional vectors $\left(b_{1}, b_{2}, \ldots, b_{k-1}, b_{k}\right)$ such that hyperplanes with the slope coefficients $\left(b_{1}, \ldots, b_{k-1}\right)$ and the location coefficient $b_{k}$ separate two groups of points in $\tilde{S}(X)$ : the class for which $P^{l} \geq 0.5$ and that for which $P^{l}<0.5$ :

$$
\forall(l=1, \ldots, d) \quad x_{1}^{l} b_{1}+x_{2}^{l} b_{2}+\ldots+x_{k-1}^{l} b_{k-1}+b_{k} \geq 0 \quad \Leftrightarrow \quad P^{l} \geq 0.5 .
$$


For each class, consider the convex hull of its points. Because the closures of the convex hulls do not intersect, there are separating hyperplanes that do not contain any points from $\tilde{S}(X)$. In other words, these hyperplanes are separated from either class by a strictly positive distance:

$$
\exists(b \in \tilde{B}) \exists(\delta>0) \forall(l=1, \ldots, d) \quad \operatorname{sgn}\left(P^{l}-0.5\right)\left(x_{1}^{l} b_{1}+x_{2}^{l} b_{2}+\ldots+x_{k-1}^{l} b_{k-1}+b_{k}\right) \geq \delta .
$$

Note that in this assertion, I have incorporated the finite support of $X$ and the constant term among regressors. Because for any $b \in \tilde{B}$, vector $\alpha b, \alpha>0$, defines the same hyperplane as $b, \delta$ can be any positive number. Without a loss of generality, suppose that $\delta=1$. Let $h$ stand for the separating hyperplane $x_{1}^{l} b_{1}+x_{2}^{l} b_{2}+\ldots+x_{k-1}^{l} b_{k-1}+b_{k}=0$. Let $h_{1}$ denote the hyperplane $x_{1}^{l} b_{1}+x_{2}^{l} b_{2}+\ldots+x_{k-1}^{l} b_{k-1}+b_{k}=1$ and $h_{2}$ denote the hyperplane $x_{1}^{l} b_{1}+x_{2}^{l} b_{2}+\ldots+x_{k-1}^{l} b_{k-1}+b_{k}=-1$. Then, according to (4.5), all points from one class lie above or lie on hyperplane $h_{1}$ and all points from the other class lie below or lie on hyperplane $h_{2}$.

If model $(B R M)$ is misspecified, then the two classes of points may be not linearly divisible. In this case, I introduce non-negative slack variables to allow for some error in separation and to find a soft margin hyperplane.

Let $v_{l} \geq 0, l=1, \ldots, d$, be slack variables. The value of $v^{l}$ is interpreted as a classification error of point $x^{l}$. Consider the following linear programming problem:

$$
\min _{b,\left\{v_{l}\right\}_{l=1}^{d}} Q(b, v)=\sum_{l=1}^{d} v_{l}
$$

subject to

$$
\begin{gathered}
\operatorname{sgn}\left(P^{l}-0.5\right)\left(x_{1}^{l} b_{1}+x_{2}^{l} b_{2}+\ldots+x_{k-1}^{l} b_{k-1}+b_{k}\right) \geq 1-v_{l}, \\
v_{l} \geq 0, \quad l=1, \ldots, d .
\end{gathered}
$$

Denote its set of solutions as $D^{*} \subset \Re^{k+d}$. Let

$B^{*}=\left\{b \in \Re^{k}:(b, v) \in D^{*}\right.$ for some $\left.v \in \Re^{d}\right\}, V^{*}=\left\{v \in \Re^{d}:(b, v) \in D^{*}\right.$ for some $\left.b \in \Re^{k}\right\}$.

Notice that when $(B R M)$ is well specified, the optimal value of the objective function is 0 , and $V^{*}=\{(0, \ldots, 0)\}$.

For any $\left(b^{*}, v^{*}\right) \in D^{*}$, a hyperplane $x_{1} b_{1}^{*}+\ldots+x_{k-1} b_{k-1}^{*}+b_{k}^{*}=0$ defined by $b^{*}$ is called a soft margin hyperplane. Because $\sum_{l=1}^{d} v_{l}$ can be interpreted as a general classification error, soft margin hyperplanes minimize this error.

Take the following example. In Figure 4, points with $P^{l} \geq 0.5$ are depicted as dark circles, and points with $P^{l}<0.5$ are depicted as white circles. As we can see, the two classes of points are not linearly separable. Consider a hyperplane $h$ defined by a vector $b$ 
(with $b_{1}>0$ ): $x_{1} b_{1}+x_{2} b_{2}+b_{3}=0$. Also picture two hyperplanes parallel to $h$ that are separated from it by an equal distance: hyperplane $h_{1}$, defined as $x_{1} b_{1}+x_{2} b_{2}+b_{3}=1$, and hyperplane $h_{2}$, defined as $x_{1} b_{1}+x_{2} b_{2}+b_{3}=-1$. In the case of separability, we could find a $b$ such that all dark points would lie above or on hyperplane $h_{1}$, and all the white points would lie below or on hyperplane $h_{2}$. From this point of view, points 7,8 and 10 are located on the correct side of $h$ and $h_{1}$. Therefore, their classification errors $v_{7}, v_{8}$ and $v_{10}$ are 0 . Point 9, on the other hand, is located on the correct side of $h$ but the incorrect side of $h_{1}$; the distance from point 9 to its correct location (that is, to $h_{1}$ ) is $v_{9}$, the point's classification error. For 6 , located on the incorrect side of $h$, the distance to $h_{1}$ is $v_{6}$. In the second class only point 3 , located on the incorrect side of $h$, has a classification error; its distance to $h_{2}$ is $v_{3}$. In fact, hyperplane $h$, as shown on Figure 4 , is a soft margin hyperplane for the depicted two classes of points.

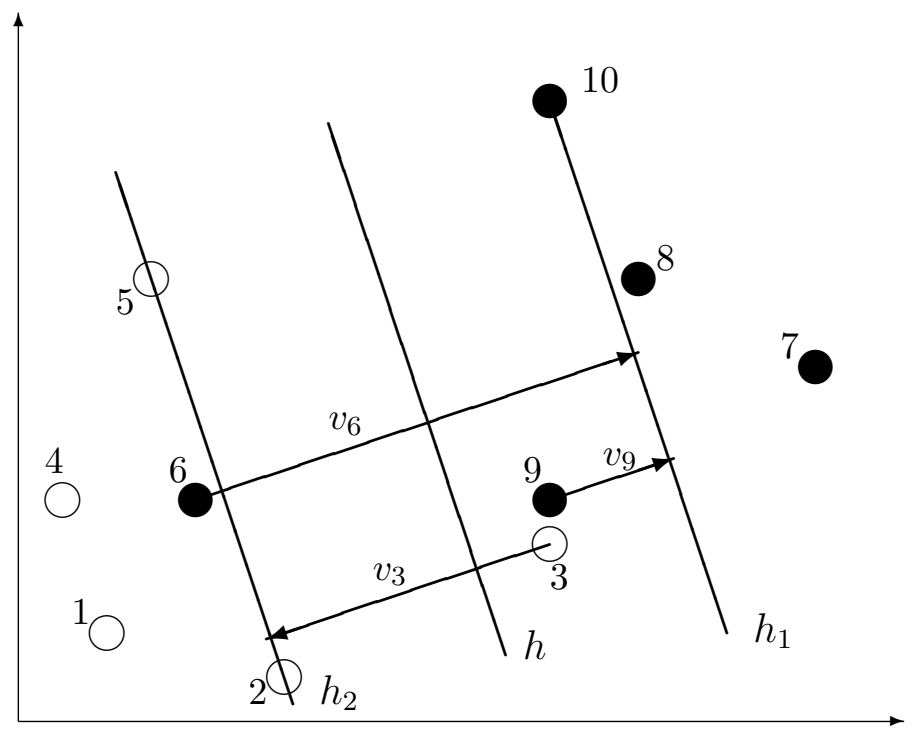

Figure 4. A soft margin separating hyperplane $(h)$ and classification errors

Let me now return to the general problem. If instead of $\delta=1$ we took a different $\delta>0$ and solved (4.6) subject to

$$
\operatorname{sgn}\left(P^{l}-0.5\right)\left(x_{1}^{l} b_{1}+x_{2}^{l} b_{2}+\ldots+x_{k-1}^{l} b_{k-1}+b_{k}\right) \geq \delta-v_{l}
$$

we would obtain the same soft margin hyperplanes, though the solution set $D^{*}(\delta)$ would differ from $D^{*}$. Namely, set $D^{*}(\delta)$ would comprise the elements of $D^{*}$ multiplied by $\delta$ : $D^{*}(\delta)=\delta D^{*}$. Given this scale effect, we are interested in the set

$$
B_{1}^{*}=\left\{\left(b_{2}, \ldots, b_{k}\right): \exists(\gamma>0) \quad\left(\gamma, \gamma b_{2}, \ldots, \gamma b_{k}\right) \in B^{*}\right\} \subset \Re^{k-1}
$$

rather than set $B^{*}$ itself. (At this stage, without a loss of generality I assume that $b_{1}^{*}>0$ for any $b^{*} \in B^{*}$.) 
Finding bounds on $B_{1}^{*}$ would be difficult. To do that, we would have to consider every solution in $B^{*}$, divide all of its coordinates by the first coordinate to find ratios, and summarize the results for all solutions.

The problem becomes much easier if we are satisfied with finding bounds for a subset of $B_{1}^{*}$. Let $\left(b^{*}, v^{*}\right)$ be any element from $D^{*}$. Define $B_{1, s}^{*} \subset \Re^{k-1}$ as the solution set $\left(b_{2}, \ldots, b_{k}\right)$ of the following system:

$$
\operatorname{sgn}\left(P^{l}-0.5\right)\left(x_{1}^{l}+x_{2}^{l} b_{2}+\ldots+x_{k-1}^{l} b_{k-1}+b_{k}\right) \geq \frac{1-v_{l}^{*}}{b_{1}^{*}}, \quad l=1, \ldots, d .
$$

Clearly, $B_{1, s}^{*} \subset B_{1}^{*}$, and system (4.7) can be rewritten in the form of $\left(S_{1}\right)$. Therefore, we can find the bounds on $B_{1, s}^{*}$ with the recursive procedure.

Let me discuss some modifications of the soft margin hyperplane method. In the general classification error in (4.6), all the slack variables have the same weight. In SVMs, all the training data points are of equal importance, therefore, SVMs consider a general classification error only in this form. Nevertheless, we can discriminate between points in $S(X)$ and assign different weights to slack variables. In other words, the general classification error can take the form $\sum_{l=1}^{d} \lambda_{l} v_{l}$, where $\sum_{l=1}^{d} \lambda_{l}=1, \lambda_{l} \geq 0, l=1, \ldots, d$. For instance, we may be willing to assign more importance to points with a higher probability of occurring and consider the objective function $\sum_{l=1}^{d} q^{l} v_{l}$. Different weights $\lambda_{l}$ will yield different solution sets $B_{1}^{*}$ and, consequently, different sets $B_{1, s}^{*}$.

In the soft margin approach in SVMs, instead of problem (3.5), the method solves the following quadratic programming problem:

$$
\min _{w_{1}, \ldots, w_{k}, a, \xi} \sum_{j=1}^{k} w_{j}^{2}+C \sum_{i=1}^{n} \xi_{i}^{\sigma}
$$

subject to

$$
\begin{gathered}
y_{i}\left[w_{1} x_{i, 1}+\ldots+w_{k} x_{i, k}-a\right] \geq 1-\xi_{i}, \\
\xi_{i} \geq 0, \quad i=1, \ldots, n
\end{gathered}
$$

for given $0<\sigma \leq 1$ and $C>0$. The objective function presents the trade-off between the maximal margin and the minimal penalty. For $\sigma=1$, the penalty function is linear in $\xi$. When $\sigma$ is small, the penalty function is close to the number of classification errors.

The idea of using linear programming problems with slack variables in the context of identification is suggested in Honore and Tamer (2006). In the authors' framework, a system of linear equations describes the identification set. To check whether a particular parameter value belongs to the set, they introduce non-negative slack variables into the equation constraints and minimize their sum subject to these modified restrictions. If the optimal function value is 0 , then the parameter value belongs to the identification set. 


\section{Dense support}

One of the sufficient conditions for point identification in $(B R)$ given by Manski (1988) is that for almost every $x_{2}, \ldots, x_{k}$ the distribution of $X_{1}$ conditional on $x_{2}, \ldots, x_{k}$, has a density positive almost everywhere. It is intuitive that when covariates are discrete but the values of $x_{1}$ corresponding to a fixed vector $\left(x_{2}, \ldots, x_{k}\right)$ form a rather dense set for many values of $\left(x_{2}, \ldots, x_{k}\right)$, then the identification set should be small. Proposition 5.1 formalizes this suggestion.

Proposition 5.1. Consider system $\left(S_{1}\right)$ with $k=3$. Suppose that its solution set is non-empty and bounded. Also, suppose that the system contains four inequalities

$$
\begin{aligned}
& z_{i_{1}, 1}+z_{i_{1}, 2} b_{2}+z_{i_{1}, 3} b_{3} \geq 0 \\
& z_{i_{2}, 1}+z_{i_{2}, 2} b_{2}+z_{i_{2}, 3} b_{3} \geq 0 \\
& z_{j_{1}, 1}+z_{j_{1}, 2} b_{2}+z_{j_{1}, 3} b_{3} \geq 0 \\
& z_{j_{2}, 1}+z_{j_{2}, 2} b_{2}+z_{j_{2}, 3} b_{3} \geq 0
\end{aligned}
$$

such that

$$
\begin{gathered}
z_{i_{1}, 2}>0, z_{i_{2}, 2}>0 \\
z_{i_{1}, 2} z_{i_{2}, 3}-z_{i_{1}, 3} z_{i_{2}, 2}>0 \\
\left(z_{j_{1}, 2}, z_{j_{1}, 3}\right)=-\left(z_{i_{1}, 2}, z_{i_{1}, 3}\right), z_{i_{1}, 1}+z_{j_{1}, 1}<\Delta \\
\left(z_{j_{2}, 2}, z_{j_{2}, 3}\right)=-\left(z_{i_{2}, 2}, z_{i_{2}, 3}\right), z_{i_{2}, 1}+z_{j_{2}, 1}<\Delta
\end{gathered}
$$

for a fixed $\Delta>0$. Then

$$
b_{3}^{u}-b_{3}^{l} \leq \frac{\left|\begin{array}{cc}
\Delta & -z_{i_{2}, 2} \\
\Delta & z_{i_{1}, 2}
\end{array}\right|}{\left|\begin{array}{ll}
z_{i_{2}, 3} & z_{i_{2}, 2} \\
z_{i_{1}, 3} & z_{i_{1}, 2}
\end{array}\right|}=\Delta \frac{\left|\begin{array}{cc}
1 & -z_{i_{2}, 2} \\
1 & z_{i_{1}, 2}
\end{array}\right|}{\left|\begin{array}{ll}
z_{i_{2}, 3} & z_{i_{2}, 2} \\
z_{i_{1}, 3} & z_{i_{1}, 2}
\end{array}\right|}
$$

where $b_{3}^{u}, b_{3}^{l}$ are defined as in Proposition 3.3.

This result is obtained using the symmetry of the formulas for $b_{3}^{l}$ and $b_{3}^{u}$. If we take four other inequalities that satisfy the conditions of the proposition, then we obtain a different bound for $b_{3}^{u}-b_{3}^{l}$, and we can choose the lower of two.

The role of Proposition 3.3 may be better appreciated if we formulate an analogous result in terms of the properties of the support.

Corollary 5.2. Let $B$ be non-empty and bounded. Suppose that there exist $\left(x_{2}, x_{3}\right)$ and $\left(x_{2}^{*}, x_{3}^{*}\right)$ such that

$$
x_{2} x_{3}^{*}-x_{2}^{*} x_{3} \neq 0, x_{2} \neq 0, x_{2}^{*} \neq 0 .
$$


Also suppose that

$$
\begin{gathered}
\exists\left(x_{1}, \tilde{x}_{1}:\left(x_{1}, x_{2}, x_{3}\right),\left(\tilde{x}_{1}, x_{2}, x_{3}\right) \in S(X)\right) \forall(b \in B) \\
x_{1}+x_{2} b_{2}+x_{3} b_{3} \geq 0, \quad \tilde{x}_{1}+x_{2} b_{2}+x_{3} b_{3}<0, \quad x_{1}-\tilde{x}_{1}<\Delta
\end{gathered}
$$

and

$$
\begin{gathered}
\exists\left(x_{1}^{*}, \tilde{x}_{1}^{*}:\left(x_{1}^{*}, x_{2}^{*}, x_{3}^{*}\right),\left(\tilde{x}_{1}^{*}, x_{2}^{*}, x_{3}^{*}\right) \in S(X)\right) \forall(b \in B) \\
x_{1}^{*}+x_{2}^{*} b_{2}+x_{3}^{*} b_{3} \geq 0, \quad \tilde{x}_{1}^{*}+x_{2}^{*} b_{2}+x_{3}^{*} b_{3}<0, \quad x_{1}^{*}-\tilde{x}_{1}^{*}<\Delta
\end{gathered}
$$

for a given $\Delta>0$. Then

$$
b_{3}^{u}-b_{3}^{l} \leq \Delta \frac{\left|x_{2}\right|+\left|x_{2}^{*}\right|}{\left|x_{2} x_{3}^{*}-x_{2}^{*} x_{3}\right|} .
$$

Proposition 5.3 is an analog of Proposition 5.1 for the case of $k=4$.

Proposition 5.3. Consider system $\left(S_{1}\right)$ with $k=4$. Let its solution set be non-empty and bounded. Suppose that $\left(S_{1}\right)$ contains eight inequalities

$$
\begin{array}{r}
z_{i_{1}, 1}+z_{i_{1}, 2} b_{2}+z_{i_{1}, 3} b_{3}+z_{i_{1}, 4} b_{4} \geq 0 \\
\ldots \\
z_{i_{4}, 1}+z_{i_{4}, 2} b_{2}+z_{i_{4}, 3} b_{3}+z_{i_{4}, 4} b_{4} \geq 0 \\
z_{j_{1}, 1}+z_{j_{1}, 2} b_{2}+z_{j_{1}, 3} b_{3}+z_{j_{1}, 4} b_{4} \geq 0 \\
\ldots \\
z_{j_{4}, 1}+z_{j_{4}, 2} b_{2}+z_{j_{4}, 3} b_{3}+z_{i_{4}, 4} b_{4} \geq 0
\end{array}
$$

such that

$$
\left(z_{j_{m}, 2}, z_{j_{m}, 3}, z_{j_{m}, 4}\right)=-\left(z_{i_{m}, 2}, z_{i_{m}, 3}, z_{i_{m}, 4}\right), \quad z_{i_{m}, 1}+z_{j_{m}, 1}<\Delta, \quad m=1,2,3,4
$$

for some $\Delta>0$, and also

$$
\begin{gathered}
z_{i_{1}, 2}>0, \quad z_{i_{2}, 2}>0, \quad z_{i_{3}, 2}>0, \quad z_{i_{4}, 2}>0, \\
z_{i_{2}, 3} z_{i_{1}, 2}-z_{i_{2}, 2} z_{i_{1}, 3}>0, \quad z_{i_{4}, 3} z_{i_{3}, 2}-z_{i_{4}, 2} z_{i_{3}, 3}>0, \\
D>0
\end{gathered}
$$

where

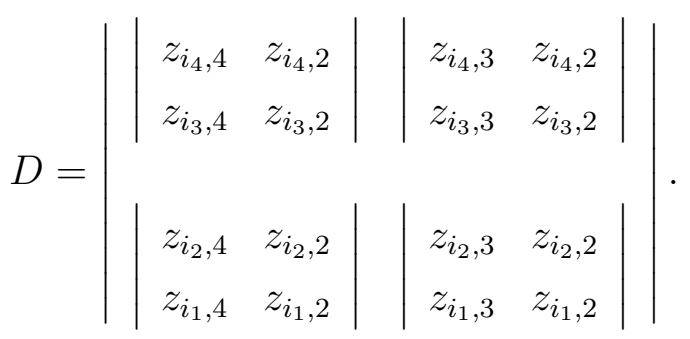


Then

$$
b_{4}^{u}-b_{4}^{l} \leq \frac{\Delta}{D}|| \begin{array}{ll}
1 & -z_{i_{4}, 2} \\
1 & z_{i_{3}, 2}
\end{array}|-| \begin{array}{ll}
z_{i_{4}, 3} & z_{i_{4}, 2} \\
z_{i_{3}, 3} & z_{i_{3}, 2}
\end{array}||,
$$

where $b_{4}^{u}, b_{4}^{l}$ are defined as in Proposition 3.4.

As in the case $k=3$ we can obtain bounds on $b_{4}^{u}-b_{4}^{l}$ in terms of the properties of the support $S(X)$. Results for $k=3$ and $k=4$ can be generalized for the case of any $k$.

\section{$6 \quad$ Asymptotic properties}

\subsection{Consistency}

The system of inequalities that defines the identification set can be constructed once it is known whether conditional probabilities $P^{l}$ are below 0.5 or not. Usually, only estimates of conditional probabilities are available, so a natural question is, how close does an estimated identification set get to the true set as the sample size increases.

In this section, I assume that model $(B R M)$ is well specified, and I distinguish two cases. In one case, all conditional probabilities are different from 0.5. In the other one, some conditional probabilities are 0.5. I differentiate these two situations because in the latter instance, the problem of finding the identification set is ill-posed in the sense that small changes in the estimators of conditional probabilities may cause considerable changes in the estimator of the identification set, no matter how large a sample size is.

Theorem 6.1. Suppose that

$$
\hat{P}_{N}^{l} \stackrel{p}{\rightarrow} P^{l} \text { as } N \rightarrow \infty
$$

and $P^{l} \neq 0.5, l=1, \ldots, d$. Let $B_{N}$ be a solution set for the system of linear inequalities derived by the rule

$$
\forall\left(x^{l} \in S(X)\right) \quad \hat{P}_{N}^{l} \geq 0.5 \Leftrightarrow x^{l} b \geq 0 .
$$

Then

$$
\operatorname{Pr}\left(B_{N} \neq B\right) \rightarrow 0 \quad \text { as } \quad N \rightarrow \infty
$$

Theorem 6.1 allows me to formulate properties of convergence in terms of Hausdorff distances. The next corollary shows that the Hausdorff distance between the estimated set and the identification set converges in probability to 0 with an arbitrary rate.

Corollary 6.2. Under the conditions of Theorem 6.1,

$$
\tau_{N} H\left(B_{N}, B\right) \stackrel{p}{\rightarrow} 0 \quad \text { as } \quad N \rightarrow \infty
$$


for any $0<\tau_{N}<\infty$. (For instance, one can take $\tau_{N}=N^{c}, c>0$.)

The statement of Theorem 6.1 does not hold if there exist $P^{l}$ equal to 0.5. Nevertheless, in this case a consistent estimator of $B$ can be derived by introducing slack variables $\epsilon_{N}$.

Theorem 6.3. Suppose that

$$
\tau_{N}\left(\hat{P}_{N}^{l}-P^{l}\right), \quad l=1, \ldots, d
$$

has a non-degenerate distribution limit as $N \rightarrow \infty$, where $0<\tau_{N}<\infty, \tau_{N}$ is increasing and $\tau_{N} \rightarrow \infty$ as $N \rightarrow \infty$. Let $\epsilon_{N}$ be a sequence of numbers such that

$$
\epsilon_{N}>0 \text { and } \epsilon_{N} \rightarrow 0, \quad \epsilon_{N} \tau_{N} \rightarrow \infty \quad \text { as } \quad N \rightarrow \infty
$$

Let $B_{N}$ be a solution set for the system of linear inequalities derived by the rule

$$
\forall\left(x^{l} \in S_{N}(X)\right) \quad \hat{P}_{N}^{l} \geq 0.5-\epsilon_{N} \quad \Leftrightarrow \quad x^{l} b \geq 0 .
$$

Then

$$
\operatorname{Pr}\left(B_{N} \neq B\right) \rightarrow 0 \quad \text { as } \quad N \rightarrow \infty
$$

Given that the support of $X$ is finite, it may be convenient to use frequency estimates of conditional probabilities:

$$
\hat{P}_{N}^{l}=\frac{\sum y_{i} 1\left(x_{i}=x^{l}\right)}{\sum 1\left(x_{i}=x^{l}\right)},
$$

based on a random sample $\left(y_{i}, x_{i}\right)_{i=1}^{N}$. These estimates allow me to prove a stronger consistency result.

Theorem 6.4. Let $\hat{P}_{N}^{l}$ be defined as in (6.5) and $B_{N}$ be the solution set for the system of linear inequalities derived by the rule (6.2).

If $P^{l} \neq 0.5$ for any $x^{l} \in S(X)$, then

$$
\exists(0<\rho<1) \quad \operatorname{Pr}\left(B_{N} \neq B\right)=o\left(\rho^{N}\right) \text { as } N \rightarrow \infty .
$$

If $P^{l}=0.5$ for some $x^{l} \in S(X)$, then asymptotically, the estimator $B_{N}$ differs from the identification set $B$ with positive probability:

$$
\exists\left(p_{0}>0\right) \quad \operatorname{Pr}\left(B_{N} \neq B\right) \geq p_{0} \text { as } N \rightarrow \infty .
$$

It is important to note that random sampling errors in estimated response probabilities may cause the system of inequalities based on $\hat{P}_{N}^{l}$ to have no solution. In this case, $B_{N}$ would be empty. One way to address this problem is to consider sample analogs of the objective functions in sections 4.1 and 4.2. For instance, for the objective function in 
section 4.2 a sample analog of the objective function is

$$
Q_{N}(b)=\sum_{l=1}^{d} \operatorname{sgn}\left(x^{l} b\right) \operatorname{sgn}\left(P_{N}^{l}-0.5\right) .
$$

Let $B_{N}^{*}$ be the set of maximizers of $Q_{N}(b)$ :

$$
B_{N}^{*}=\left\{\left(b_{2}, \ldots, b_{k}\right):\left(1, b_{2}, \ldots, b_{k}\right) \in \operatorname{Argmax}_{b: b_{1}=1} Q_{N}(b)\right\}
$$

If $B_{N} \neq \emptyset$, then $B_{N}=B_{N}^{*}$. All results in the current section remain true if we substitute $B_{N}$ for $B_{N}^{*}$. Other objective functions $Q_{N}(\cdot)$ also can be considered. Because the model is well specified, the only condition required for $Q_{N}(\cdot)$ is that the set of maximizers of its population analog $Q(\cdot)$ coincides with $B$. In general, it is preferable to consider objective functions that reflect some of the model's properties.

If $B_{N}=\emptyset$, either sampling errors in conditional probabilities estimates or misspecification could be at fault. Though it would be interesting to develop tests to distinguish between those two cases, I leave this task to future research.

Let me analyze the behavior of the maximum score estimates obtained from a random sample. In the sample, the maximum score estimates maximize the function

$$
S_{N}^{m s}(b)=\frac{1}{N} \sum_{i=1}^{N}\left(2 y_{i}-1\right) \operatorname{sgn}\left(x_{i} b\right),
$$

which can be equivalently written as

$$
S_{N}^{m s}(b)=2 \sum_{l=1}^{d} \hat{q}_{N}^{l}\left(\hat{P}_{N}^{l}-0.5\right) \operatorname{sgn}\left(x^{l} b\right),
$$

where $\hat{P}_{N}^{l}$ is defined as in (6.5).

Proposition 6.5. Suppose that model (BRM) is well specified. Let

$$
B_{N}^{m s}=\left\{\left(b_{2}, \ldots, b_{k}\right):\left(1, b_{2}, \ldots, b_{k}\right) \in \operatorname{Argmax}_{b: b_{1}=1} S_{N}^{m s}(b)\right\} .
$$

If $P^{l} \neq 0.5$ for any $x^{l} \in S(X)$, then

$$
\exists(0<\rho<1) \quad \operatorname{Pr}\left(B_{N}^{m s} \neq B\right)=o\left(\rho^{N}\right) \text { as } N \rightarrow \infty .
$$

If $P^{l}=0.5$ for some $x^{l} \in S(X)$, then

$$
\exists\left(p_{0}>0\right) \quad \operatorname{Pr}\left(B_{N}^{m s} \neq B\right) \geq p_{0} \text { as } N \rightarrow \infty
$$




\subsection{Statistical inference}

I now describe two methods of building confidence regions for the identification set. Both are based on the normal approximations of conditional probabilities $P^{l}$. Because of the problem's discrete nature, we cannot always achieve an exact nominal confidence level; in many cases, the true confidence coefficient is greater than the stated level.

Given a random sample of size $N$, the objective is to construct regions $B_{N}$ that asymptotically cover $B$ with probability $1-\alpha$, where $\alpha$ is a prespecified value between 0 and 1:

$$
\lim _{N \rightarrow \infty} P\left(B_{N} \supset B\right) \geq 1-\alpha .
$$

Let $\hat{P}_{N}^{l}$ be a frequency estimator of $P^{l}$ as in (6.5). Asymptotically,

$$
\sqrt{N}\left(\hat{P}_{N}^{l}-P^{l}\right) \rightarrow N\left(0,\left(\sigma^{l}\right)^{2}\right), \quad \text { where } \quad\left(\sigma^{l}\right)^{2}=\frac{P^{l}\left(1-P^{l}\right)}{q^{l}}
$$

Substitute $\sigma^{l}$ with its estimate $\hat{\sigma}_{N}^{l}$ :

$$
\sqrt{N} \frac{\hat{P}_{N}^{l}-P^{l}}{\hat{\sigma}_{N}^{l}} \rightarrow N(0,1), \quad \text { where } \quad \hat{\sigma}_{N}^{l}=\frac{\hat{P}_{N}^{l}\left(1-\hat{P}_{N}^{l}\right)}{\hat{q}_{N}^{l}} .
$$

In the first method, I choose numbers $\left\{\gamma_{l}\right\}_{l=1}^{d}$ such that $\gamma_{l} \geq 0, l=1, \ldots, d$, and $\sum_{l=1}^{d} \gamma_{l}=\alpha$. Let $\zeta_{\gamma_{l}}$ denote the $1-\gamma_{l}$ quantile of the standard normal distribution, then construct a system of linear inequalities in the following way:

if for a given $x^{l}$

$$
\hat{P}_{N}^{l}-\zeta_{\gamma_{l}} \frac{\hat{\sigma}_{N}^{l}}{\sqrt{N}}>0.5
$$

then add the inequality $x^{l} b \geq 0$ to the system. If

$$
\hat{P}_{N}^{l}+\zeta_{\gamma_{l}} \frac{\hat{\sigma}_{N}^{l}}{\sqrt{N}}<0.5
$$

then add the inequality $-x^{l} b>0$ to the system. If the interval $\left[\hat{P}_{N}^{l}-\zeta_{\gamma_{l}} \frac{\hat{\sigma}_{N}^{l}}{\sqrt{N}}, \hat{P}_{N}^{l}+\zeta_{\gamma_{l}} \frac{\hat{\sigma}_{N}^{l}}{\sqrt{N}}\right]$ contains 0.5 , then no inequalities in the system correspond to $x^{l}$. I claim that the solution set $B_{N}$ for the system constructed according to this method has the property

$$
P\left(B_{N} \supset B\right) \rightarrow 1 \text { as } N \rightarrow \infty
$$

Indeed,

$P\left(B_{N} \not \supset B\right) \leq \sum_{l=1}^{d}\left(P\left(\hat{P}_{N}^{l}-\zeta_{\gamma_{l}} \frac{\hat{\sigma}_{N}^{l}}{\sqrt{N}}>0.5 \mid P^{l}<0.5\right)+P\left(\hat{P}_{N}^{l}-\zeta_{\gamma_{l}} \frac{\hat{\sigma}_{N}^{l}}{\sqrt{N}}<0.5 \mid P^{l}>0.5\right)\right)$. 
Notice that

$$
P\left(\hat{P}_{N}^{l}-\zeta_{\gamma_{l}} \frac{\hat{\sigma}_{N}^{l}}{\sqrt{N}}>0.5 \mid P^{l}<0.5\right)=1-\Phi\left(\left(0.5-P^{l}\right) \frac{\sqrt{N}}{\hat{\sigma}_{N}^{l}}-\zeta_{\gamma_{l}}\right) \rightarrow 0 \text { as } N \rightarrow \infty
$$

and, similarly,

$$
P\left(\hat{P}_{N}^{l}-\zeta_{\gamma_{l}} \frac{\hat{\sigma}_{N}^{l}}{\sqrt{N}}<0.5 \mid P^{l}>0.5\right) \rightarrow 0 \text { as } N \rightarrow \infty
$$

Clearly,

$$
P\left(B_{N} \supset B\right)=1-P\left(B_{N} \not \supset B\right) \rightarrow 1 \text { as } N \rightarrow \infty .
$$

As we can see, confidence region $B_{N}$ is overly conservative: Its actual coverage probability is 1 . In this sense, it is inaccurate.

Furthermore, in practice set $B_{N}$ can be empty. In this case, instead of $B_{N}$, we can find a set $B_{N}^{*}$ that solves optimization problem

$$
\max _{b: b_{1}=1} \sum_{l=1}^{d} \operatorname{sgn}\left(x^{l} b \geq 0\right)\left(1\left(\hat{P}_{N}^{l}-\zeta_{\gamma_{l}} \frac{\hat{\sigma}_{N}^{l}}{\sqrt{N}}>0.5\right)-1\left(\hat{P}_{N}^{l}+\zeta_{\gamma_{l}} \frac{\hat{\sigma}_{N}^{l}}{\sqrt{N}}<0.5\right)\right) .
$$

If $B_{N}$ is not empty, $B_{N}=B_{N}^{*}$. Using the technique I employed for region $B_{N}$, I can prove that $\operatorname{Pr}\left(B_{N}^{*} \supset B\right) \rightarrow 1$ as $N \rightarrow \infty$.

The method of finding $B_{N}^{*}$ is equivalent to the following approach. Let

$$
I=\prod_{l=1}^{d}\left[\hat{P}_{N}^{l}-\zeta_{\gamma_{l}} \frac{\hat{\sigma}_{N}^{l}}{\sqrt{N}}, \hat{P}_{N}^{l}+\zeta_{\gamma_{l}} \frac{\hat{\sigma}_{N}^{l}}{\sqrt{N}}\right]
$$

Set $I$ is an asymptotic $1-\alpha$ confidence set for $P=\left(P^{1}, \ldots, P^{d}\right)$. If for any $\tilde{P}=$ $\left(\tilde{P}^{1}, \ldots, \tilde{P}^{d}\right) \in I$ we construct a system of inequalities by the rule

$$
\tilde{P}_{l} \geq 0.5 \quad \Leftrightarrow \quad x^{l} b \geq 0
$$

and find its solution $B_{N, \tilde{P}}$, then we can show that

$$
B_{N}^{*}=\cup_{\tilde{P} \in I} B_{N, \tilde{P}}
$$

(Of course, many of sets $B_{N, \tilde{P}}$ will be empty.)

Let me describe another method for building confidence sets. Note that asymptotically

$$
\sum_{l=1}^{d} N \frac{\left(\hat{P}_{N}^{l}-P^{l}\right)^{2}}{\left(\hat{\sigma}_{N}^{l}\right)^{2}} \rightarrow \chi^{2}(d-1) \quad \text { as } \quad N \rightarrow \infty
$$


Instead of $I$, consider ellipsoid $E$, which is a $1-\alpha$ confidence set for $P$ :

$$
E=\left\{P=\left(\tilde{P}^{1}, \ldots, \tilde{P}^{d}\right): \sum_{l=1}^{d} \frac{\left(\hat{P}_{N}^{l}-\tilde{P}^{l}\right)^{2}}{\left(\hat{\sigma}_{N}^{l}\right)^{2}} \leq \frac{\chi_{1-\alpha}^{2}(d-1)}{N}\right\}
$$

If for any $\tilde{P}=\left(\tilde{P}^{1}, \ldots, \tilde{P}^{d}\right) \in E$ we construct a system of inequalities according to (6.7) and find its solution set $B_{N, \tilde{P}}$, then region

$$
B_{N, E}^{*}=\cup_{\tilde{P} \in E} B_{N, \tilde{P}}
$$

satisfies (6.6).

Recent studies on the construction of confidence sets for partially identified parameters include Imbens and Manski (2004), Chernozhukov, Hong and Tamer (2007) and Rosen (2006), among others. Imbens and Manski (2004) propose confidence intervals that cover the true value of the parameter rather than the entire identification region. Chernozhukov, Hong and Tamer (2007) consider models in which the identification region is the set of the minimizers for a criterion function. They build confidence regions with a specified probability by using a suggested subsampling procedure. Rosen (2006) examines models defined by a finite number of moment inequalities and constructs confidence sets through pointwise testing. In recent years, there has also been increasing interest in finite-sample methods of inference. For instance, for binary choice and multinomial choice models, Manski (2007) develops confidence sets that are valid for all sample sizes.

\section{$7 \quad$ Empirical example}

\subsection{Monte Carlo simulations}

The design of the Monte Carlo experiment is based on Example 3.1. The outcome data are generated as follows:

$$
Y=1\left(X_{1}+1.25 X_{2}-0.5 X_{3}+U \geq 0\right)
$$

$X_{1}, X_{2}$ and $X_{3}$ take the values outlined in Example 3.1 ( $X_{2}$ is the constant term). I specify distributions for $X_{1}$ and $X_{3}$. Let the distribution of the error term be

$$
U \mid x \sim \frac{x_{1} 1\left(x_{1}<0\right)}{\sqrt{2 x_{1}^{2}+2 x_{3}^{2}+0.001}} Z+0.1 x_{3} 1\left(x_{1} \geq 0\right) V,
$$

where random variable $Z$ has a standard normal distribution, random variable $V$ is distributed uniformly on $[-1,1]$ and $Z$ and $V$ are independent. I report results for a sample of size $N=5,000$. Note that the conditional median independence assumption is satisfied. 
For comparison, I apply several estimation procedures. There are 87 points in the sample's support. From the sample I calculate frequency estimates of conditional probabilities. Using these estimates I construct a system of linear inequalities by the rule

$$
\hat{P}_{N}^{l} \geq 0.5 \quad \Leftrightarrow \quad x_{1}^{l}+b_{2}+b_{3} x_{3}^{l} \geq 0
$$

and apply the recursive procedure to find bounds on $\beta_{2}$ and $\beta_{3}$. Because the system has solutions, its set of maximum score estimates coincides with its solution set. See Table 1 for the estimation results. Observe that the set of maximum rank correlation estimates for $\beta_{3}$ does not contain value -0.5 used to design the experiment, although this value is very close to its border.

I want to emphasize that the results presented for the recursive procedure and the maximum score method are identification intervals for each individual parameter; the identification set for $\left(\beta_{2}, \beta_{3}\right)$ is smaller than rectangle $(1,1.6) \times(-0.6,-0.42587)$ (see Figure 2).

I also report probit and logit estimates with $95 \%$ confidence intervals for each parameter, as well as normalized probit and logit estimates (ratios $\hat{\beta}_{2} / \hat{\beta}_{1}$ and $\hat{\beta}_{3} / \hat{\beta}_{1}$ ). As we can see, the normalized probit and logit estimates belong to the identification intervals, but they are far from the parameter values used to generate the outcome data.

\begin{tabular}{lccc}
\hline \hline & $X_{1}$ & $X_{2}=$ CONST & $X_{3}$ \\
\hline Recursive procedure & 1 & $\mathbf{( 1 , ~ 1 . 6 )}$ & $\mathbf{( - 0 . 6 , - 0 . 4 2 5 8 7 )}$ \\
$\begin{array}{l}\text { Set of maximum score } \\
\text { estimates }\end{array}$ & 1 & $\mathbf{( 1 , ~ 1 . 6 )}$ & $\mathbf{( - 0 . 6 , - 0 . 4 2 5 8 7 )}$ \\
MRC & 1 & & \\
Probit & 2.9152 & 4.3893 & $\mathbf{( - 0 . 5 7 1 4 , - 0 . 5 0 0 1 )}$ \\
& $(2.3794,3.4510)$ & $(3.4141,5.3646)$ & -1.5922 \\
Probit (r) & 1 & $\mathbf{1 . 5 0 5 7}$ & $(-1.9173,-1.2672)$ \\
& & $(1.1711,1.8402)$ & $(-0.6577,-0.4347)$ \\
Logit & 5.4318 & 8.0505 & -2.9515 \\
& $(4.3201,6.5435)$ & $(6.0992,10.0019)$ & $(-3.6126,-2.2904)$ \\
Logit (r) & 1 & $\mathbf{1 . 4 8 2 1}$ & $\mathbf{- 0 . 5 4 3 4}$ \\
& & $(1.1229,1.8414)$ & $(-0.6651,-0.4217)$ \\
\hline
\end{tabular}

Table 1. Estimation results for the Monte Carlo experiment 


\subsection{Women's labor force participation}

In this section I present an empirical application based on MROZ data regarding married women's labor force participation (WORK). Let $W O R K=1$ if a woman participates in the labor force; otherwise, let $W O R K=0$. The variables we use to explain labor force participation are education (EDUC), experience (EXPER), age (AGE) and number of children under six years old (KIDS). The descriptive statistics for these variables are presented in Table 2 .

\begin{tabular}{lcccc}
\hline \hline & EDUC & EXPER & AGE & KIDS \\
\hline Mean & 12.287 & 10.631 & 42.538 & 0.238 \\
SD & 2.280 & 8.069 & 8.073 & 0.524 \\
Median & 12 & 9 & 43 & 0 \\
Min. & 5 & 0 & 30 & 0 \\
Max. & 17 & 45 & 60 & 3 \\
\hline
\end{tabular}

Table 2. Descriptive statistics for MROZ data

Thus, we estimate the binary response model

$$
W O R K_{i}=1\left(E D U C_{i}+\beta_{0}+\beta_{E X P E R} E X P E R_{i}+\beta_{A G E} A G E_{i}+\beta_{K I D S} K I D S_{i}+u_{i} \geq 0\right),
$$

where I normalize the coefficient corresponding to EDUC. For comparison, I apply several estimation procedures. Table 3 contains the results of these estimations.

There are $N=753$ observations. After calculating frequency estimates $\hat{P}_{N}^{l}$ and combining data for women with identical characteristics, I obtain 670 points in the support. Based on $\hat{P}_{N}^{l}$, I construct a system of inequalities as usual. This system has no solution, so I employ the methods suggested in section 4 for dealing with misspecification.

MS stands for the maximum score estimation methods; the set of maximum score estimates is the union of several disjoint convex polyhedra, and the reported bounds are the sharp bounds for this union. MNCE stands for the method of minimal number of classification errors described in section 4.2; the set of MNCE estimates is the union of several disjoint convex polyhedra, and the reported bounds are the sharp bounds for that union. MGCE stands for the minimal general classification error method outlined in section 4.3. The set of MGCE estimates is a convex polyhedron. MRC stands for the maximum rank correlation method; the set of maximum rank correlation estimates is the union of several disjoint convex polyhedra (in $\Re^{3}$ ), and the bounds shown in Table 3 are the sharp bounds for this union.

I also include normalized probit, logit, OLS and LAD estimates (ratios $\hat{\beta}_{0} / \hat{\beta}_{E D U C}$, 


\begin{tabular}{lcccc}
\hline \hline & CONST & EXPER & AGE & KIDS \\
\hline MS & $(2.5972,2.7714)$ & $(0.92361,0.9433)$ & $(-0.48571,-0.47917)$ & $(-5.1429,-4.9931)$ \\
MNCE & $(7.3514,7.8447)$ & $(1.0412,1.0651)$ & $(-0.608,-0.59513)$ & $(-12.536,-8.7)$ \\
MGCE & $(6.9065,6.9224)$ & $(0.63395,0.63427)$ & $(-0.51237,-0.51201)$ & $(-7.5439,-7.5389)$ \\
MRC & & $(0.83343,0.85997)$ & $(-0.59998,-0.58065)$ & $(-8.7897,-8.5005)$ \\
Probit(r) & 7.68301 & 0.65254 & -0.54431 & -7.86264 \\
& $(0.0189,15.3471)$ & $(0.5247,0.7804)$ & $(-0.6799,-0.4088)$ & $(-9.8915,-5.8338)$ \\
Logit(r) & 6.96944 & 0.65890 & -0.53036 & -7.68174 \\
& $(-0.6815,14.6203)$ & $(0.5215,0.7963)$ & $(-0.6686,-0.3921)$ & $(-9.7471,-5.6164)$ \\
OLS(r) & 23.47147 & 0.68967 & -0.57315 & -8.20569 \\
& $(15.6655,31.2775)$ & $(0.5134,0.7311)$ & $(-0.6355,-0.3987)$ & $(-9.1492,-5.6577)$ \\
LAD(r) & 26.53767 & 0.78495 & -0.68817 & -10.40862 \\
& $(17.1571,35.9182)$ & $(0.6402,0.9297)$ & $(-0.8457,-0.5306)$ & $(-12.7282,-8.089)$ \\
\hline
\end{tabular}

Table 3. Estimation of labor force participation

$\hat{\beta}_{E X P E R} / \hat{\beta}_{E D U C}, \hat{\beta}_{A G E} / \hat{\beta}_{E D U C}$ and $\left.\hat{\beta}_{K I D S} / \hat{\beta}_{E D U C}\right)$.

As we can see, the results produced by methods MS, MNCE, MGCE and MRC are in certain sense consistent with each other. For each regressor, the method MGCE provides shorter intervals than methods MS, MNCE and MRC. This does not come as a surprise, because, first of all, MGCE finds only a subset of separating hyperplanes, and, second, it can be shown that this subset always lies in a hyperplane in the space $\Re^{k-1}$ (in our case, in the space $R^{4}$ ).

\section{Conclusion}

In this paper, I examine binary response models when the regressors have discrete support. Ignoring the continuity conditions sufficient for point identification can lead to unsound and misleading inference results on the parameter of interest.

Given these concerns, it is critical to seek a complete characterization of the parameters that fit the model. This paper provides such a characterization for semiparametric binary response models. I offer a recursive procedure to find the sharp bounds on the parameter's identification set. A big advantage of this procedure is the ease of implementation. Moreover, it allows us to explore other aspects of identification, such as the extrapolation problem or changes in the identification set when one regressor's support becomes increasingly dense. Furthermore, the procedure can be used in single-index models with a monotone link function and in ordered-response models. 
I go beyond the identification issue by investigating the estimation of the identification region and examining model's misspecification, which I approach in several different ways and provide insight into its possible causes and consequences. I also present an empirical application that compares several estimation techniques and argue that the results critically depend on our preferences for a certain estimation approach.

Several unresolved issues would benefit from future research. It is interesting to look deeper into model's misspecification. Studies that develop tests for misspecification would be particularly useful. When the identification set estimated from a random sample is empty, for instance, we would like to have a test that would allow us to determine whether misspecification or random sampling are behind this problem. Another worthwhile extension would be to learn how to construct finite-sample confidence sets for the identification region.

Despite the several issues that remain to be explored, this paper enhances our understanding of the structure and properties of the identification region in binary response models with discrete regressors. It also provides empirical economists with another avenue for using semiparametric methods when data do not satisfy the sufficient conditions for point identification. 


\section{$9 \quad$ Appendix}

\subsection{Examples of the recursive procedure}

Example 9.1. Consider the following system of inequalities with three unknown variables:

$$
\begin{aligned}
-b_{2}+3 b_{3}-4 b_{4} & \geq 0 \\
4-b_{2} & \geq 0 \\
2+b_{2}-2 b_{3}+6 b_{4} & \geq 0 \\
b_{2}+2 b_{4} & \geq 0 \\
-1-b_{2}-5 b_{4} & \geq 0 .
\end{aligned}
$$

To eliminate variable $b_{2}$ from this system, rewrite it as

$$
\begin{array}{r}
3 b_{3}-4 b_{4} \geq b_{2} \\
4 \geq b_{2} \\
b_{2} \geq-2+2 b_{3}-6 b_{4} \\
b_{2} \geq-2 b_{4} \\
-1-5 b_{4} \geq b_{2}
\end{array}
$$

and obtain that

$$
\begin{array}{r}
3 b_{3}-4 b_{4} \geq-2+2 b_{3}-6 b_{4} \\
3 b_{3}-4 b_{4} \geq-2 b_{4} \\
4 \geq-2+2 b_{3}-6 b_{4} \\
4 \geq-2 b_{4} \\
-1-5 b_{4} \geq-2+2 b_{3}-6 b_{4} \\
-1-5 b_{4} \geq-2 b_{4},
\end{array}
$$

or, equivalently,

$$
\begin{aligned}
2+b_{3}+2 b_{4} & \geq 0 \\
6-2 b_{3}+6 b_{4} & \geq 0 \\
1-2 b_{3}+b_{4} & \geq 0 \\
3 b_{3}-2 b_{4} & \geq 0 \\
4+2 b_{4} & \geq 0 \\
-1-3 b_{4} & \geq 0 .
\end{aligned}
$$


Example 9.2. Exclude $b_{3}$ from (9.2) and obtain the following system:

$$
\begin{aligned}
10+10 b_{4} & \geq 0 \\
5+5 b_{4} & \geq 0 \\
18+14 b_{4} & \geq 0 \\
3-b_{4} & \geq 0 \\
4+2 b_{4} & \geq 0 \\
-1-3 b_{4} & \geq 0 .
\end{aligned}
$$

From system (9.3), find that $\underline{b}_{4}=-1$ and $\bar{b}_{4}=-1 / 3$. Similarly, find sharp bounds on $b_{2}$ by excluding $b_{3}$ and $b_{4}$ from the system: $\underline{b}_{2}=2 / 3$ and $\bar{b}_{2}=4$. For $b_{3}$, find that $\underline{b}_{3}=-1 / 2$ and $\bar{b}_{3}=1 / 3$.

Example 9.3. Add to system (9.1) one more inequality:

$$
-6-b_{2}+4 b_{3}+10 b_{4} \geq 0
$$

Then there will be two more inequalities in system (9.2):

$$
\begin{aligned}
& -4+2 b_{3}+16 b_{4} \geq 0 \\
& -6+4 b_{3}+5 b_{4} \geq 0,
\end{aligned}
$$

After eliminating $b_{3}$ obtain system (9.3) plus two more inequalities

$$
\begin{gathered}
2+22 b_{4} \geq 0 \\
-2+7 b_{4} \geq 0,
\end{gathered}
$$

and find that $\underline{b}_{4}=2 / 7$ and $\bar{b}_{4}=-1 / 3$. Because $\underline{b}_{4}>\bar{b}_{4}$, the system has no solution.

Example 9.4. Add to system (9.1) one more inequality:

$$
-5-b_{2}+2 b_{3}-6 b_{4} \geq 0
$$

Then there will be two more inequalities in system (9.2):

$$
\begin{aligned}
-3 & \geq 0 \\
-6+4 b_{3}+12 b_{4} & \geq 0 .
\end{aligned}
$$

There is an obvious contradiction $-3 \geq 0$ in the system, so it does not have solutions.

Example 9.5. Drawing from system (9.1), we modify two inequalities and consider the following 
system:

$$
\begin{aligned}
-b_{2}+3 b_{3}-4 b_{4} & \geq 0 \\
4-b_{2}+4 b_{3} & \geq 0 \\
2+b_{2}-2 b_{3}+6 b_{4} & \geq 0 \\
b_{2}+2 b_{4} & \geq 0 \\
-1-b_{2}+5 b_{3}-5 b_{4} & \geq 0 .
\end{aligned}
$$

Eliminating variable $b_{2}$ from (9.4), we obtain

$$
\begin{aligned}
2+b_{3}+2 b_{4} & \geq 0 \\
3 b_{3}-2 b_{4} & \geq 0 \\
6+2 b_{3}+6 b_{4} & \geq 0 \\
4+4 b_{3}+2 b_{4} & \geq 0 \\
1+3 b_{3}+b_{4} & \geq 0 \\
-1+5 b_{3}-3 b_{4} & \geq 0 .
\end{aligned}
$$

All coefficients corresponding to $b_{3}$ are positive, indicating not only that the system has solutions but also that values of variable $b_{3}$ are not bounded from above. Although $b_{3}$ cannot be eliminated from the system, $b_{4}$ has coefficients of both signs and therefore can be excluded to obtain information about $b_{3}$. After eliminating $b_{4}$, we will obtain eight inequalities, from which we will find that $\underline{b}_{3}=-1 / 7$.

If we excluded $b_{3}(9.4)$ at the first step, we would obtain the following system:

$$
\begin{aligned}
6+3 b_{2}+10 b_{4} & \geq 0 \\
8+b_{2}+12 b_{4} & \geq 0 \\
8+3 b_{2}+20 b_{4} & \geq 0 \\
b_{2}+2 b_{4} & \geq 0 .
\end{aligned}
$$

Because all coefficients corresponding to $b_{2}$ and $b_{3}$ would be positive, we would conclude that $b_{2}$ and $b_{3}$ are bounded from neither below nor above.

\subsection{Proofs}

\section{Proof of Proposition 3.1.}

Even though an analog of this proposition is established in Solodovnikov (1977), it is worth proving it here. The first part is intuitive, so I focus on the second claim. Let $\left(b_{3}^{*}, \ldots, b_{k}^{*}\right)$ be a solution of $\left(S_{2}\right)$. Plug these numbers into $D_{i}$ and $N_{j}$ and obtain numbers $D_{i}^{*}$ and $N_{j}^{*}$ such that

$$
N_{j}^{*} \geq D_{i}^{*}, \quad i=1, \ldots, I, \quad j=1, \ldots, J
$$


Take any $b_{2}$ such that

$$
\min _{j=1, \ldots, J} N_{j}^{*} \geq b_{2} \geq \max _{i=1, \ldots, I} D_{i}^{*},
$$

then $\left(b_{2}, b_{3}^{*}, \ldots, b_{k}^{*}\right)$ is a solution of $\left(S_{1}\right)$.

\section{Proof of Proposition 3.3}

In the system

$$
\begin{array}{r}
z_{11}+z_{12} b_{2}+z_{13} b_{3} \geq 0 \\
\ldots \\
z_{d 1}+z_{d 2} b_{2}+z_{d 3} b_{3} \geq 0
\end{array}
$$

consider any inequality

$$
z_{j 1}+z_{j 2} b_{2}+z_{j 3} b_{3} \geq 0
$$

with $z_{j 2}<0$. This inequality is equivalent to

$$
-\frac{z_{j 1}}{z_{j 2}}-\frac{z_{j 3}}{z_{j 2}} b_{3} \geq b_{2} .
$$

Now consider any inequality

$$
z_{i 1}+z_{i 2} b_{2}+z_{i 3} b_{3} \geq 0,
$$

with $z_{i 2}>0$ and rewrite it as

$$
b_{2} \geq-\frac{z_{i 1}}{z_{i 2}}-\frac{z_{i 3}}{z_{i 2}} b_{3} .
$$

Necessarily,

$$
-\frac{z_{j 1}}{z_{j 2}}-\frac{z_{j 3}}{z_{j 2}} b_{3} \geq-\frac{z_{i 1}}{z_{i 2}}-\frac{z_{i 3}}{z_{i 2}} b_{3}
$$

that is,

$$
\frac{z_{i 1}}{z_{i 2}}-\frac{z_{j 1}}{z_{j 2}} \geq\left(\frac{z_{j 3}}{z_{j 2}}-\frac{z_{i 3}}{z_{i 2}}\right) b_{3} .
$$

If

$$
\frac{z_{j 3}}{z_{j 2}}-\frac{z_{i 3}}{z_{i 2}}>0
$$

then

$$
b_{3} \leq \frac{\frac{z_{i 1}}{z_{i 2}}-\frac{z_{j 1}}{z_{j 2}}}{\frac{z_{j 3}}{z_{j 2}}-\frac{z_{i 3}}{z_{i 2}}}=\frac{z_{i 1} z_{j 2}-z_{j 1} z_{i 2}}{z_{j 3} z_{i 2}-z_{i 3} z_{j 2}}=-\frac{\left|\begin{array}{cc}
z_{j 1} & z_{j 2} \\
z_{i 1} & z_{i 2}
\end{array}\right|}{\left|\begin{array}{cc}
z_{j 3} & z_{j 2} \\
z_{i 3} & z_{i 2}
\end{array}\right|}
$$

where

$$
\left|\begin{array}{cc}
z_{j 3} & z_{j 2} \\
z_{i 3} & z_{i 2}
\end{array}\right|=z_{i 2} z_{j 2}\left(\frac{z_{j 3}}{z_{j 2}}-\frac{z_{i 3}}{z_{i 2}}\right)<0 .
$$

Because (9.5) holds for an arbitrary $i$ and $j$ such that $z_{j 2}<0, z_{i 2}>0$ and (9.6) are satisfied, 
then

$$
b_{3} \leq \min _{i, j}\left\{-\frac{\left|\begin{array}{cc}
z_{j 1} & z_{j 2} \\
z_{i 1} & z_{i 2}
\end{array}\right|}{\left|\begin{array}{cc}
z_{j 3} & z_{j 2}<0 \\
z_{i 3} & z_{i 2}>0
\end{array}\right|<0}\right\} .
$$

Similarly, prove that

$$
b_{3} \geq-\frac{\left|\begin{array}{cc}
z_{j 1} & z_{j 2} \\
z_{i 1} & z_{i 2}
\end{array}\right|}{\left|\begin{array}{cc}
z_{j 3} & z_{j 2} \\
z_{i 3} & z_{i 2}
\end{array}\right|}
$$

for any $i$ and $j$ such that $z_{j 2}<0, z_{i 2}>0$ and

$$
\left|\begin{array}{cc}
z_{j 3} & z_{j 2} \\
z_{i 3} & z_{i 2}
\end{array}\right|=z_{i 2} z_{j 2}\left(\frac{z_{j 3}}{z_{j 2}}-\frac{z_{i 3}}{z_{i 2}}\right)>0
$$

that is,

$$
b_{3} \geq \max _{i, j}\left\{-\frac{\left|\begin{array}{cc}
z_{j 1} & z_{j 2} \\
z_{i 1} & z_{i 2}
\end{array}\right|}{\left|\begin{array}{cc}
z_{j 3} & z_{j 2}<0 \\
z_{i 3} & z_{i 2}>0
\end{array}\right|>0}\right\} .
$$

\section{Proof of Proposition 3.5}

The proof proceeds by induction on $k$. As has been proved above, this proposition holds for $k=3$. Suppose that it also holds for some value $k$. For this case, let us prove that it holds for $k+1$ as well. Consider system

$$
\begin{array}{r}
z_{11}+z_{12} b_{2}+\ldots+z_{1 k} b_{k}+z_{1, k+1} b_{k+1} \geq 0 \\
z_{21}+z_{22} b_{2}+\ldots+z_{2 k} b_{k}+z_{2, k+1} b_{k+1} \geq 0 \\
\ldots \\
z_{n 1}+z_{n 2} b_{2}+\ldots+z_{n k} b_{k}+z_{n, k+1} b_{k+1} \geq 0
\end{array}
$$

and apply the recursive algorithm to exclude $b_{2}$ from the system. The new system consists of inequalities of the form

$$
\left(\frac{z_{i 1}}{z_{i 2}}-\frac{z_{j 1}}{z_{j 2}}\right)+\left(\frac{z_{i 3}}{z_{i 2}}-\frac{z_{j 3}}{z_{j 2}}\right) b_{3}+\ldots+\left(\frac{z_{i, k+1}}{z_{i 2}}-\frac{z_{j, k+1}}{z_{j 2}}\right) b_{k+1} \geq 0
$$

where $z_{i 2}>0$ and $z_{j 2}<0$. Let us write this system as

$$
r_{l 1}+r_{l 3} b_{3}+\ldots+r_{l, k+1} b_{k+1} \geq 0, \quad l=1, \ldots, n_{1} .
$$


Let $\tilde{A}_{d}, d \geq 1$ stand for the determinants corresponding to this new system and $A_{d}$ stand for the determinants corresponding to the original system. Let us show that $\tilde{A}_{d}$ is determined by $2^{d+1}$ indices $i_{1}, j_{1}, \ldots, i_{2^{d}}, j_{2^{d}}$ and that

$$
\tilde{A}_{d}\left(m, i_{1}, j_{1}, \ldots, i_{2^{d}}, j_{2^{d}}\right)=\frac{1}{z_{i_{1} 2} z_{j_{1} 2} \ldots z_{i_{2^{d}}} z_{j_{2^{d}}}} A_{d+1}\left(m+1, i_{1}, j_{1}, \ldots, i_{2^{d}}, j_{2^{d}}\right) .
$$

To prove this, use the induction method. Consider $d=1$ :

$$
\tilde{A}_{1}\left(m, l_{1}, l_{2}\right)=\left|\begin{array}{cc}
r_{l_{2}, m+1} & r_{l_{2}, 3} \\
r_{l_{1}, m+1} & r_{l_{1}, 3}
\end{array}\right|
$$

Inequality $l_{1}$ was obtained from some inequalities $i_{1}$ and $j_{1}$ of the original system. Similarly, inequality $l_{2}$ has some corresponding inequalities $i_{2}$ and $j_{2}$. Then

$$
\begin{aligned}
& \tilde{A}_{1}\left(m, l_{1}, l_{2}\right)=\tilde{A}_{1}\left(m, i_{1}, j_{1}, i_{2}, j_{2}\right)=\left|\begin{array}{cc}
r_{l_{2}, m+1} & r_{l_{2}, 3} \\
r_{l_{1}, m+1} & r_{l_{1}, 3}
\end{array}\right|= \\
& =\frac{1}{z_{i_{1} 2} z_{j_{1} 2} z_{i_{2} 2} z_{j_{2} 2}}|| \begin{array}{cc}
z_{j_{1} m+1} & z_{j_{1} 2} \\
z_{i_{1} m+1} & z_{i_{1} 2}
\end{array}|| \begin{array}{cc}
z_{j_{1} 3} & z_{j_{1} 2} \\
z_{i_{1} 3} & z_{i_{1} 2}
\end{array}||= \\
& =\frac{1}{z_{i_{1} 2} z_{j_{1}} z_{i_{2} 2} z_{j_{2} 2}}\left|\begin{array}{ll}
A_{1}\left(m+1, i_{1}, j_{1}\right) & A_{1}\left(3, i_{1}, j_{1}\right) \\
A_{1}\left(m+1, i_{2}, j_{2}\right) & A_{1}\left(3, i_{2}, j_{2}\right)
\end{array}\right| \text {. }
\end{aligned}
$$

Thus, for $d=1$ the statement is true. Suppose that it is also true for some $d-1$. Let us prove that in this case, it is also true for $d$. Because $\tilde{A}_{d-1}$ depends on $2^{d-1}$ indices, then

$$
\tilde{A}_{d}(m, \ldots,)=\left|\begin{array}{cl}
\tilde{A}_{d-1}\left(m, i_{1}, \ldots, j_{2^{d-1}}\right) & \tilde{A}_{d-1}\left(k+1, i_{1}, \ldots, j_{2^{d-1}}\right)<0 \\
\tilde{A}_{d-1}\left(m, i_{2^{d-1}+1}, \ldots, j_{2^{d}}\right) & \tilde{A}_{d-1}\left(k+1, i_{2^{d-1}+1}, \ldots, j_{2^{d}}\right)>0
\end{array}\right|
$$

depends on $2^{d}$ indices. For $d-1$, the statement of the lemma is true. Therefore,

$$
\begin{aligned}
& \tilde{A}_{d}\left(m, i_{1}, \ldots, j_{2^{d}}\right)= \\
& =\frac{1}{z_{i_{1} 2 \ldots j_{2^{d-1}}{ }^{2} i_{2^{d-1}+1^{2}}{ }^{2 \ldots} z_{2^{d^{d}}}}}\left|\begin{array}{cl}
A_{d}\left(m+1, i_{1}, \ldots, j_{2^{d-1}}\right) & A_{d}\left(d+2, i_{1}, \ldots, j_{2^{d-1}}\right)<0 \\
A_{d}\left(m+1, i_{2^{d-1}+1}, \ldots, j_{2^{d}}\right) & A_{d}\left(d+2, i_{2^{d-1}+1}, \ldots, j_{2^{d}}\right)>0
\end{array}\right|=
\end{aligned}
$$

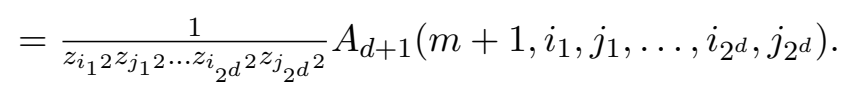

Because

$$
\frac{\tilde{A}_{k-2}\left(1, i_{1}, \ldots, j_{2^{k-2}}\right)}{\tilde{A}_{k-2}\left(k, i_{1}, \ldots, j_{2^{k-2}}\right)}=\frac{A_{k-1}\left(1, i_{1}, \ldots, j_{2^{k-2}}\right)}{A_{k-1}\left(k+1, i_{1}, \ldots, j_{2^{k-2}}\right)}
$$

then we conclude that the formula is true for $b_{k+1}$. 
The maximal possible value of $S^{m s}$ is $2 \sum_{x^{l} \in S(X)} q^{l}\left|P^{l}-0.5\right|$. Evidently, this value is attained on set $B$; that is, $B \subseteq B^{m s}$. On the other hand, if $P^{l} \neq 0.5$ for any $x^{l} \in S(X)$, then $\max _{b: b_{1}=1} S^{m s}(b)=2 \sum_{x^{l} \in S(X)} q^{l}\left|P^{l}-0.5\right|$ implies that there exists $b$ that solves the system of linear inequalities constructed according to the rule

$$
P^{l} \geq 0.5 \quad \Leftrightarrow \quad x^{l} b \geq 0, \quad l=1, \ldots, d,
$$

which, in turn, defines set $B$. Thus, $B^{m s} \subseteq B$, and, therefore, $B=B^{m s}$.

Now suppose that $P^{l}=0.5$ for some $l$. If, for instance, $P^{1}=0.5$, then any $b$ satisfying the system of inequalities

$$
P^{l} \geq 0.5 \quad \Leftrightarrow \quad x^{l} b \geq 0, \quad l=2, \ldots, d
$$

also gives a maximal value to $S^{m s}$. So, in this case, set $B^{m s}$ is larger than $B$; that is, $B \subset B^{m s}$.

\section{Proof of Proposition 5.1}

This proof is based on the symmetrical property of the formulas for $b_{3}^{l}$ and $b_{3}^{u}$ in Proposition 3.3. According to these formulas,

$$
\begin{gathered}
b_{3}^{u} \leq-\frac{\left|\begin{array}{ll}
z_{j_{2}, 1} & z_{j_{2}, 2} \\
z_{i_{1}, 1} & z_{i_{1}, 2}
\end{array}\right|}{\left|\begin{array}{ll}
z_{j_{2}, 3} & z_{j_{2}, 2} \\
z_{i_{1}, 3} & z_{i_{1}, 2}
\end{array}\right|}=\frac{\left|\begin{array}{ll}
z_{j_{2}, 1} & -z_{i_{2}, 2} \\
z_{i_{1}, 1} & z_{i_{1}, 2}
\end{array}\right|}{\left|\begin{array}{ll}
z_{i_{2}, 3} & z_{i_{2}, 2} \\
z_{i_{1}, 3} & z_{i_{1}, 2}
\end{array}\right|}, \\
b_{3}^{l} \geq-\frac{\left|\begin{array}{ll}
z_{j_{1}, 1} & z_{j_{1}, 2} \\
z_{i_{2}, 1} & z_{i_{2}, 2}
\end{array}\right|}{\left|\begin{array}{ll}
z_{j_{1}, 3} & z_{j_{1}, 2} \\
z_{i_{2}, 3} & z_{i_{2}, 2}
\end{array}\right|}=-\frac{\left|\begin{array}{ll}
z_{j_{1}, 1} & -z_{i_{1}, 2} \\
z_{i_{2}, 1} & z_{i_{2}, 2}
\end{array}\right|}{\left|\begin{array}{ll}
z_{i_{2}, 3} & z_{i_{2}, 2} \\
z_{i_{1}, 3} & z_{i_{1}, 2}
\end{array}\right|}
\end{gathered}
$$

and, hence,

$$
\begin{aligned}
& b_{3}^{u}-b_{3}^{l} \leq \frac{\left|\begin{array}{cc}
z_{j_{1}, 1} & -z_{i_{1}, 2} \\
z_{i_{2}, 1} & z_{i_{2}, 2}
\end{array}\right|+\left|\begin{array}{cc}
z_{j_{2}, 1} & -z_{i_{2}, 2} \\
z_{i_{1}, 1} & z_{i_{1}, 2}
\end{array}\right|}{\left|\begin{array}{ll}
z_{i_{2}, 3} & z_{i_{2}, 2} \\
z_{i_{1}, 3} & z_{i_{1}, 2}
\end{array}\right|}=\frac{z_{i_{2}, 2}\left(z_{j_{1}, 1}+z_{i_{1}, 1}\right)+z_{i_{1}, 2}\left(z_{j_{2}, 1}+z_{i_{2}, 1}\right)}{\left|\begin{array}{ll}
z_{i_{2}, 3} & z_{i_{2}, 2} \\
z_{i_{1}, 3} & z_{i_{1}, 2}
\end{array}\right|} \leq \\
& \leq \frac{\Delta\left(z_{i_{2}, 2}+z_{i_{1}, 2}\right)}{\left|\begin{array}{lll}
z_{i_{2}, 3} & z_{i_{2}, 2} \\
z_{i_{1}, 3} & z_{i_{1}, 2}
\end{array}\right|}=\Delta \frac{\left|\begin{array}{cc}
1 & -z_{i_{2}, 2} \\
1 & z_{i_{1}, 2}
\end{array}\right|}{\left|\begin{array}{cc}
z_{i_{2}, 3} & z_{i_{2}, 2} \\
z_{i_{1}, 3} & z_{i_{1}, 2}
\end{array}\right|} .
\end{aligned}
$$

\section{Proof of Corollary 5.2}

Suppose that $x_{2} x_{3}^{*}-x_{2}^{*} x_{3}>0$, and consider the following four cases. 
Case 1: $x_{2}>0, x_{2}^{*}>0$. Define

$$
\begin{array}{ll}
\left(z_{i_{1}, 1}, z_{i_{1}, 2}, z_{i_{1}, 3}\right)=\left(x_{1}, x_{2}, x_{3}\right), & \left(z_{j_{1}, 1}, z_{j_{1}, 2}, z_{j_{1}, 3}\right)=\left(-\tilde{x}_{1},-x_{2},-x_{3}\right), \\
\left(z_{i_{2}, 1}, z_{i_{2}, 2}, z_{i_{2}, 3}\right)=\left(x_{1}^{*}, x_{2}^{*}, x_{3}^{*}\right), & \left(z_{j_{2}, 1}, z_{j_{2}, 2}, z_{j_{2}, 3}\right)=\left(-\tilde{x}_{1}^{*},-x_{2}^{*},-x_{3}^{*}\right) .
\end{array}
$$

Then all conditions in Proposition 5.1 are satisfied. Therefore,

$$
b_{3}^{u}-b_{3}^{l} \leq \Delta \frac{x_{2}+x_{2}^{*}}{x_{2} x_{3}^{*}-x_{2}^{*} x_{3}}
$$

Case 2: $x_{2}>0, x_{2}^{*}<0$. Define

$$
\begin{aligned}
& \left(z_{i_{1}, 1}, z_{i_{1}, 2}, z_{i_{1}, 3}\right)=\left(-\tilde{x}_{1}^{*},-x_{2}^{*},-x_{3}^{*}\right), \quad\left(z_{j_{1}, 1}, z_{j_{1}, 2}, z_{j_{1}, 3}\right)=\left(x_{1}^{*}, x_{2}^{*}, x_{3}^{*}\right) \\
& \left(z_{i_{2}, 1}, z_{i_{2}, 2}, z_{i_{2}, 3}\right)=\left(x_{1}, x_{2}, x_{3}\right), \quad\left(z_{j_{2}, 1}, z_{j_{2}, 2}, z_{j_{2}, 3}\right)=\left(-\tilde{x}_{1},-x_{2},-x_{3}\right)
\end{aligned}
$$

Then all condition in Proposition 5.1 are satisfied. Therefore,

$$
b_{3}^{u}-b_{3}^{l} \leq \Delta \frac{x_{2}-x_{2}^{*}}{x_{2} x_{3}^{*}-x_{2}^{*} x_{3}}
$$

Case 3: $x_{2}<0, x_{2}^{*}>0$. Define

$$
\begin{aligned}
& \left(z_{i_{1}, 1}, z_{i_{1}, 2}, z_{i_{1}, 3}\right)=\left(x_{1}^{*}, x_{2}^{*}, x_{3}^{*}\right), \quad\left(z_{j_{1}, 1}, z_{j_{1}, 2}, z_{j_{1}, 3}\right)=\left(-\tilde{x}_{1}^{*},-x_{2}^{*},-x_{3}^{*}\right), \\
& \left(z_{i_{2}, 1}, z_{i_{2}, 2}, z_{i_{2}, 3}\right)=\left(-\tilde{x}_{1},-x_{2},-x_{3}\right), \quad\left(z_{j_{2}, 1}, z_{j_{2}, 2}, z_{j_{2}, 3}\right)=\left(x_{1}, x_{2}, x_{3}\right) .
\end{aligned}
$$

Then all condition in Proposition 5.1 are satisfied. Therefore,

$$
b_{3}^{u}-b_{3}^{l} \leq \Delta \frac{-x_{2}+x_{2}^{*}}{x_{2} x_{3}^{*}-x_{2}^{*} x_{3}}
$$

Case 3: $x_{2}<0, x_{2}^{*}<0$. Define

$$
\begin{array}{r}
\left(z_{i_{1}, 1}, z_{i_{2}, 2}, z_{i_{2}, 3}\right)=\left(-\tilde{x}_{1},-x_{2},-x_{3}\right), \quad\left(z_{j_{1}, 1}, z_{j_{1}, 2}, z_{j_{1}, 3}\right)=\left(x_{1}, x_{2}, x_{3}\right), \\
\left(z_{i_{2}, 1}, z_{i_{2}, 2}, z_{i_{2}, 3}\right)=\left(-\tilde{x}_{1}^{*},-x_{2}^{*},-x_{3}^{*}\right),\left(z_{j_{2}, 1}, z_{j_{2}, 2}, z_{j_{2}, 3}\right)=\left(x_{1}^{*}, x_{2}^{*}, x_{3}^{*}\right) .
\end{array}
$$

Then all condition in Proposition 5.1 are satisfied. Therefore,

$$
b_{3}^{u}-b_{3}^{l} \leq \Delta \frac{-x_{2}-x_{2}^{*}}{x_{2} x_{3}^{*}-x_{2}^{*} x_{3}} .
$$

The case in which $x_{2} x_{3}^{*}-x_{2}^{*} x_{3}<0$ can be considered in a similar way.

\section{Proof of Proposition 5.3}

The proof of Proposition 5.3 is based on the symmetrical property of the formulas for $b_{4}^{l}$ and 
$b_{4}^{u}$. According to the formulas in Proposition 3.4,

$$
b_{4}^{l} \geq-\frac{C_{1}}{D}, \quad b_{4}^{u} \leq \frac{C_{2}}{D},
$$

where

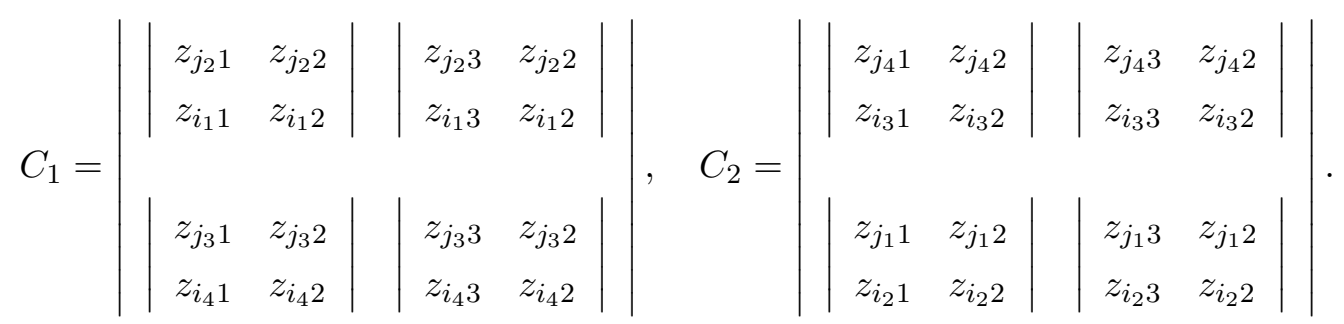

Then

$$
\begin{aligned}
& b_{4}^{u}-b_{4}^{l} \leq \frac{C_{1}}{D}+\frac{C_{2}}{D}=\frac{1}{D}\left|\begin{array}{cc}
z_{j_{4}} & z_{j_{4} 2} \\
z_{i_{3} 1} & z_{i_{3} 2}
\end{array}\right| \cdot\left|\begin{array}{cc}
z_{i_{2} 2} & z_{i_{2} 3} \\
z_{j_{1} 2} & z_{j_{1} 3}
\end{array}\right|-\frac{1}{D}\left|\begin{array}{cc}
z_{i_{2} 1} & z_{i_{2} 2} \\
z_{j_{1} 1} & z_{j_{1} 2}
\end{array}\right| \cdot\left|\begin{array}{cc}
z_{j_{4} 2} & z_{j_{4} 3} \\
z_{i_{3} 2} & z_{i_{3} 3}
\end{array}\right|+ \\
& +\frac{1}{D}\left|\begin{array}{cc}
z_{j_{2} 1} & z_{j_{2} 2} \\
z_{i_{1} 1} & z_{i_{1} 2}
\end{array}\right| \cdot\left|\begin{array}{cc}
z_{i_{4} 2} & z_{i_{4} 3} \\
z_{j_{3} 2} & z_{j_{3} 3}
\end{array}\right|-\frac{1}{D}\left|\begin{array}{cc}
z_{i_{1} 1} & z_{i_{4} 2} \\
z_{j_{3} 1} & z_{j_{3} 2}
\end{array}\right| \cdot\left|\begin{array}{cc}
z_{j_{2} 2} & z_{j_{2} 3} \\
z_{i_{1} 2} & z_{i_{1} 3}
\end{array}\right|= \\
& =\frac{1}{D}\left(z_{i_{1} 2}\left(z_{j_{2} 1}+z_{i_{2} 1}\right)+z_{i_{2} 2}\left(z_{j_{1} 1}+z_{i_{1} 1}\right)\right)\left|\begin{array}{cc}
z_{i_{4} 2} & z_{i_{4} 3} \\
z_{j_{3} 2} & z_{j_{3} 3}
\end{array}\right|+\frac{1}{D}\left(z_{i_{3} 2}\left(z_{j_{4} 1}+z_{i_{4} 1}\right)+z_{i_{4} 2}\left(z_{j_{3} 1}+z_{i_{3} 1}\right)\right)\left|\begin{array}{cc}
z_{j_{2} 2} & z_{j_{2} 3} \\
z_{i_{1} 2} & z_{i_{1} 3}
\end{array}\right| \leq
\end{aligned}
$$

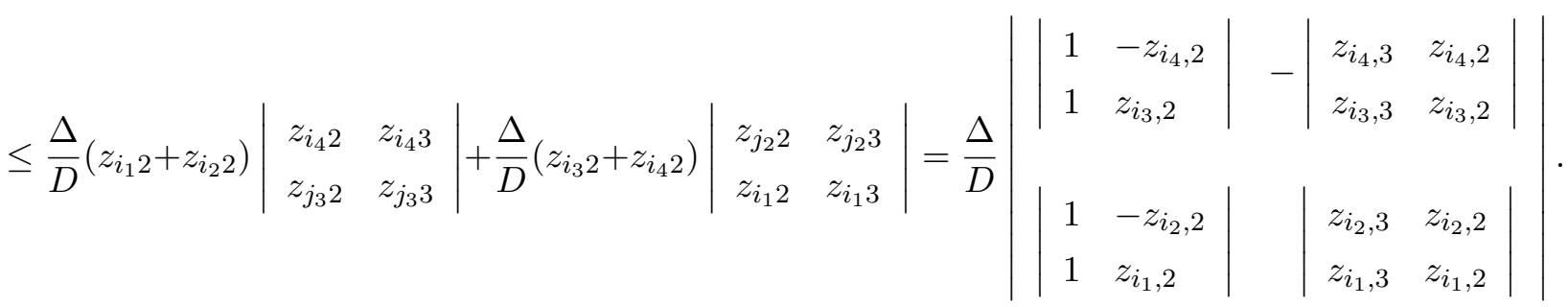

\section{Proof of Theorem 6.1}

$$
B_{N} \neq B \quad \Rightarrow \quad \exists\left(x^{l} \in S(X)\right) \quad \operatorname{sgn}\left(\hat{P}_{N}^{l}-0.5\right) \neq \operatorname{sgn}\left(P^{l}-0.5\right),
$$

therefore,

$$
\operatorname{Pr}\left(B_{N} \neq B\right) \leq \sum_{x^{l} \in S(X)} P\left(\operatorname{sgn}\left(\hat{P}_{N}^{l}-0.5\right) \neq \operatorname{sgn}\left(P^{l}-0.5\right)\right) .
$$

Because

$$
\begin{aligned}
& P^{l}>0.5 \quad \text { and } \operatorname{sgn}\left(\hat{P}_{N}^{l}-0.5\right) \neq \operatorname{sgn}\left(P^{l}-0.5\right) \quad \Rightarrow \quad P^{l}-\hat{P}_{N}^{l}>P^{l}-0.5 \text {, } \\
& P^{l}<0.5 \quad \text { and } \operatorname{sgn}\left(\hat{P}_{N}^{l}-0.5\right) \neq \operatorname{sgn}\left(P^{l}-0.5\right) \quad \Rightarrow \quad \hat{P}_{N}^{l}-P^{l}>0.5-P^{l} \text {, }
\end{aligned}
$$

then

$$
\forall\left(x^{l} \in S(X)\right) \quad \operatorname{Pr}\left(\operatorname{sgn}\left(\hat{P}_{N}^{l}-0.5\right) \neq \operatorname{sgn}\left(P^{l}-0.5\right)\right) \leq \operatorname{Pr}\left(\left|\hat{P}_{N}^{l}-P^{l}\right|>\left|0.5-P^{l}\right|\right) .
$$


The consistency property (6.1) and the fact that $\left|0.5-P^{l}\right|>0$ for any $x^{l} \in S(X)$ imply

$$
\forall\left(x^{l} \in S(X)\right) \quad \operatorname{Pr}\left(\left|\hat{P}_{N}^{l}-P^{l}\right|>\left|0.5-P^{l}\right|\right) \rightarrow 0 \text { as } N \rightarrow \infty .
$$

It is evident now that

$$
\operatorname{Pr}\left(B_{N} \neq B\right) \rightarrow 0 \text { as } N \rightarrow \infty
$$

\section{Proof of Corollary 6.2.}

For any $\epsilon>0$,

$$
\tau_{N} H\left(B_{N}, B\right) \geq \epsilon \quad \Rightarrow \quad H\left(B_{N}, B\right) \neq 0 \quad \Rightarrow \quad B_{N} \neq B
$$

Therefore,

$$
\operatorname{Pr}\left(\tau_{N} H\left(B_{N}, B\right) \geq \epsilon\right) \leq \operatorname{Pr}\left(B_{N} \neq B\right) \rightarrow 0 \quad \text { as } \quad N \rightarrow \infty
$$

\section{Proof of Theorem 6.3}

$$
\operatorname{Pr}\left(B_{N} \neq B\right) \leq \sum_{x^{l} \in S(X)} P\left(\operatorname{sgn}\left(\hat{P}_{N}^{l}-0.5+\epsilon_{N}\right) \neq \operatorname{sgn}\left(P^{l}-0.5\right)\right) .
$$

If $P^{l}>0.5$, then

$$
\begin{array}{r}
\operatorname{Pr}\left(\operatorname{sgn}\left(\hat{P}_{N}^{l}-0.5+\epsilon_{N}\right) \neq \operatorname{sgn}\left(P^{l}-0.5\right)\right)=\operatorname{Pr}\left(P^{l}-\hat{P}_{N}^{l}>P^{l}-0.5+\epsilon_{N}\right) \leq \\
\leq \operatorname{Pr}\left(P^{l}-\hat{P}_{N}^{l}>P^{l}-0.5\right) \rightarrow 0 \text { as } N \rightarrow \infty .
\end{array}
$$

Let $P^{l}<0.5$. Convergence $\epsilon_{N} \rightarrow 0$ implies that, when $N$ is large enough, $0.5-P^{l}-\epsilon_{N}>\delta$ for some $\delta>0$, and, consequently,

$$
\begin{array}{r}
\operatorname{Pr}\left(\operatorname{sgn}\left(\hat{P}_{N}^{l}-0.5+\epsilon_{N}\right) \neq \operatorname{sgn}\left(P^{l}-0.5\right)\right)=\operatorname{Pr}\left(\hat{P}_{N}^{l}-P^{l}>0.5-P^{l}-\epsilon_{N}\right) \leq \\
\leq \operatorname{Pr}\left(\hat{P}_{N}^{l}-P^{l}>\delta\right) \rightarrow 0 \quad \text { as } \quad N \rightarrow \infty .
\end{array}
$$

If $P^{l}=0.5$, then

$$
\operatorname{Pr}\left(\operatorname{sgn}\left(\hat{P}_{N}^{l}-0.5+\epsilon_{N}\right) \neq \operatorname{sgn}\left(P^{l}-0.5\right)\right)=\operatorname{Pr}\left(P^{l}-\hat{P}_{N}^{l}>\epsilon_{N}\right)=\operatorname{Pr}\left(\epsilon_{N}^{-1}\left(P^{l}-\hat{P}_{N}^{l}\right)>1\right) .
$$

(6.3) and (6.4) imply that

$$
\epsilon_{N}^{-1}\left(P^{l}-\hat{P}_{N}^{l}\right)=\left(\epsilon_{N} \tau_{N}\right)^{-1} \tau_{N}\left(P^{l}-\hat{P}_{N}^{l}\right) \stackrel{p}{\rightarrow} 0 \text { as } N \rightarrow \infty
$$

and, thus,

$$
\operatorname{Pr}\left(\epsilon_{N}^{-1}\left(P^{l}-\hat{P}_{N}^{l}\right)>1\right) \rightarrow 0 \quad \text { as } \quad N \rightarrow \infty .
$$

\section{Proof of Theorem 6.4}

$$
\operatorname{Pr}\left(B_{N} \neq B\right) \leq \sum_{x^{l} \in S(X)} \operatorname{Pr}\left(\operatorname{sgn}\left(\hat{P}_{N}^{l}-0.5\right) \neq \operatorname{sgn}\left(P^{l}-0.5\right)\right)
$$


Denote

$$
V_{N}\left(x^{l}\right)=\sum_{i=1}^{N}\left(2 y_{i}-1\right) 1\left(x_{i}=x^{l}\right) .
$$

Then

$$
\hat{P}_{N}^{l} \geq 0.5 \quad \Leftrightarrow \quad V_{N}\left(x^{l}\right) \geq 0 .
$$

Note that random variable $\left(2 y_{i}-1\right) 1\left(x_{i}=x^{l}\right)$ takes values 1,0 and -1 with probabilities $P^{l} q^{l}$, $1-q^{l}$ and $\left(1-P^{l}\right) q^{l}$, respectively. Its expected value is $\left(2 P^{l}-1\right) q^{l}$.

Let $P^{l}>0.5$. Then $\operatorname{Pr}\left(\operatorname{sgn}\left(\hat{P}_{N}^{l}-0.5\right) \neq \operatorname{sgn}\left(P^{l}-0.5\right)\right)=\operatorname{Pr}\left(V_{N}\left(x^{l}\right)<0\right)$. By Hoeffding's inequality,

$$
\operatorname{Pr}\left(V_{N}\left(x^{l}\right)<0\right)=\operatorname{Pr}\left(V_{N}\left(x^{l}\right)-N\left(2 P^{l}-1\right) q^{l}<-N\left(2 P^{l}-1\right) q^{l}\right) \leq e^{-N\left(\left(2 P^{l}-1\right) q^{l}\right)^{2} / 2} .
$$

If $P^{l}<0.5$, then $\operatorname{Pr}\left(\operatorname{sgn}\left(\hat{P}_{N}^{l}-0.5\right) \neq \operatorname{sgn}\left(P^{l}-0.5\right)\right)=\operatorname{Pr}\left(V_{N}\left(x^{l}\right) \geq 0\right)$. By Hoeffding's inequality

$$
\operatorname{Pr}\left(V_{N}\left(x^{l}\right) \geq 0\right)=\operatorname{Pr}\left(V_{N}\left(x^{l}\right)-N\left(2 P^{l}-1\right) q^{l} \geq N\left(1-2 P^{l}\right) q^{l}\right) \leq e^{-N\left(\left(2 P^{l}-1\right) q^{l}\right)^{2} / 2} .
$$

Thus, if $P^{l} \neq 0.5$,

$$
\operatorname{Pr}\left(\operatorname{sgn}\left(\hat{P}_{N}^{l}-0.5\right) \neq \operatorname{sgn}\left(P^{l}-0.5\right)\right) \leq e^{-N\left(\left(2 P^{l}-1\right) q^{l}\right)^{2} / 2} .
$$

Let $\rho<1$ be such that

$$
\rho>\max _{l=1, \ldots, d} e^{-\left(\left(2 P^{l}-1\right) q^{l}\right)^{2} / 2}
$$

Then, if $P^{l} \neq 0.5$ for any $x^{l}$,

$$
\operatorname{Pr}\left(B_{N} \neq B\right)=o\left(\rho^{N}\right) \text { as } N \rightarrow \infty
$$

This proves the first part of the theorem.

Now suppose that there is $x^{l}$ such that $P^{l}=0.5$. Because

$$
P^{l}=0.5 \text { and } P_{N}^{l}<0.5 \quad \Rightarrow \quad B_{N} \neq B
$$

then

$$
\operatorname{Pr}\left(B_{N} \neq B\right) \geq \operatorname{Pr}\left(V_{N}\left(x^{l}\right)<0\right)
$$

Note that

$$
P^{l}=0.5 \quad \Rightarrow \quad \operatorname{Pr}\left(V_{N}\left(x^{l}\right)<0\right)=0.5\left(1-\operatorname{Pr}\left(V_{N}\left(x^{l}\right)=0\right)\right) .
$$

If we will find a bound on $\operatorname{Pr}\left(V_{N}\left(x^{l}\right)=0\right)$ from above, we will find a bound on $\operatorname{Pr}\left(V_{N}\left(x^{l}\right)<0\right)$ 
from below.

$$
\begin{array}{r}
\operatorname{Pr}\left(V_{N}\left(x^{l}\right)=0\right)=\sum_{j=0}^{\left[\frac{N}{2}\right]} C_{j}^{N} C_{j}^{N-j} 0.5^{2 j}\left(q^{l}\right)^{2 j}\left(1-q^{l}\right)^{N-2 j}=\sum_{j=0}^{\left[\frac{N}{2}\right]} C_{2 j}^{N} C_{j}^{2 j} 0.5^{2 j}\left(q^{l}\right)^{2 j}\left(1-q^{l}\right)^{N-2 j}= \\
=\left(1-q^{l}\right)^{N}+\sum_{j=1}^{\left[\frac{N}{2}\right]} C_{2 j}^{N} C_{j}^{2 j} 0.5^{2 j}\left(q^{l}\right)^{2 j}\left(1-q^{l}\right)^{N-2 j} .
\end{array}
$$

Use the fact that for $j \geq 1$,

$$
C_{j}^{2 j} 0.5^{2 j}=(-1)^{j} \frac{(-0.5)(-0.5-1) \ldots(-0.5-j+1)}{j !} \leq 0.5
$$

to obtain

$$
\operatorname{Pr}\left(V_{N}\left(x^{l}\right)=0\right) \leq\left(1-q^{l}\right)^{N}+0.5 \sum_{j=1}^{\left[\frac{N}{2}\right]} C_{2 j}^{N}\left(q^{l}\right)^{2 j}\left(1-q^{l}\right)^{N-2 j} \leq\left(1-q^{l}\right)^{N}+0.5 .
$$

Then

$$
\operatorname{Pr}\left(V_{N}\left(x^{l}\right)<0\right)=0.5\left(1-\operatorname{Pr}\left(V_{N}\left(x^{l}\right)=0\right)\right) \geq 0.5\left(1-\left(1-q^{l}\right)^{N}-0.5\right)
$$

and

$$
\operatorname{Pr}\left(B_{N} \neq B\right) \geq 0.5\left(1-\left(1-q^{l}\right)^{N}-0.5\right) \rightarrow 0.25 \text { as } N \rightarrow \infty .
$$

\section{Proof of Proposition 6.5}

Suppose that $P^{l} \neq 0.5$ for any $x^{l} \in S(X)$. Then

$$
\operatorname{Pr}\left(B_{N}^{m s} \neq B\right) \leq \sum_{x^{l} \in S(X)} \operatorname{Pr}\left(\operatorname{sgn}\left(\hat{P}_{N}^{l}-0.5\right) \neq \operatorname{sgn}\left(P^{l}-0.5\right)\right)
$$

and the proof proceeds in the same way as the proof of the first part of Proposition 6.4.

The proof of the second part, when $P^{l}=0.5$ for some $x^{l} \in S(X)$, is the same as the proof of the second part of Proposition 6.4. 


\section{References}

[1] Balinski, M.L. (1961). An Algorithm for Finding All Vertices of Convex Polyhedral Sets, J. Soc. Indust. and Appl. Math., 9, 72-88.

[2] Bierens, H.J., and J. Hartog (1988). Non-Linear Regression with Explanatory Variables, with an Application to the Earnings Function, Journal of Econometrics, 38, 269-299.

[3] Cavanagh, C., and R.P. Sherman (1998). Rank Estimators for Monotonic Index Models, Journal of Econometrics, 84, 351-381.

[4] Chernikova, N.V. (1965). Algorithm for Finding a General Formula for the Nonnegative Solutions of a System of Linear Inequalities, U.S.S.R. Computational Mathematics and Mathematical Physics, 5, 228-233.

[5] Chernikova, N.V. (1965). Algorithm for Finding a General Formula for the Nonnegative Solutions of a System of Linear Inequalities, U.S.S.R. Computational Mathematics and Mathematical Physics, 5, 228-233.

[6] Chernozhukov, V., H. Hong, and E. Tamer (2007). Estimation and Confidence Regions for Parameter Sets in Econometric Models, Econometrica, 75 (5), 1243-1284.

[7] Cortes, C., and V. Vapnik (1995). Support Vector Networks, Machine Learning, 20, $1-25$.

[8] Cosslett, S.R. (1983). Distribution-Free Maximum Likelihood Estimator of the Binary Choice Model, Econometrica, 51, 765-782.

[9] Han, A.K. (1987). Non-parametric Analysis of a Generalized Regression Model, Journal of Econometrics, 35, 303-316.

[10] Honore, Bo E., and E. Tamer (2006). Bounds on Parameters in Panel Dynamic Discrete Choice Models, Econometrica, 74 (3), 611-629.

[11] Horowitz, J.L. (1992). A Smoothed Maximum Score Estimator for the Binary Response Model, Econometrica, 60, 505-531.

[12] Horowitz, J.L. (1998). Semiparametric Methods in Econometrics, Lecture Notes in Statistics, Vol. 131: Springer-Verlag New York, Inc.

[13] Ichimura, H. (1993). Semiparametric Least Squares (SLS) and Weighted SLS Estimator of Single-Index Models, Journal of Econometrics, 58, 71-120.

[14] Imbens, G.W., and C.F. Manski (2004). Confidence Intervals for Partially Identified Parameters, Econometrica, 72 (6), 1845-1857. 
[15] Kim, J., and D. Pollard (1990). Cube Root Asymptotics, Annals of Statistics, 18, 191-219.

[16] Klein, R.W., and R.H. Spady (1993). An Efficient Semiparametric Estimator of Binary Response Models, Econometrica, 61, 387-421.

[17] Kuhn, H. W. (1956). Solvability and Consistency for Linear Equations and Inequalities, The American Mathematical Monthly, 63 (4), 217-232.

[18] Kuhn, H.W. and A.W. Tucker (1956). Linear Inequalities and Related Systems, Annals of Mathematics Studies 38, Princeton U. Press, Princeton.

[19] Lin, Y. (2000). On the Support Vector Machine, Technical Report No. 1029, University of Wisconsin.

[20] Lin, Y. (2002). Support Vector Machines and the Bayes Rule in Classification, Data Miniing and Knowledge Discovery, 6 (3), 259-275.

[21] Magnac, T., and E. Maurin (2005). Partial Identification in Monotone Binary Models: Discrete Regressors and Interval Data, Working Paper, University of Toulouse.

[22] Manas, M., and J. Nedoma (1968). Finding All Vertices of a Convex Polyhedron, Numerische Mathematik, 12, 226-229.

[23] Manski, C.F. (1975). Maximum Score Estimation of the Stochastic Utility Model of Choice, Journal of Econometrics, 3, 205-228.

[24] Manski, C.F. (1985). Semiparametric Analysis of Discrete Response: Asymptotic Properties of the Maximum Score Estimator, Journal of Econometrics, 27, 313-334.

[25] Manski, C.F. (1988). Identification of Binary Response Models, Journal of the American Statistical Association, 83, No. 403, 729-738.

[26] Manski, C.F. (1990). Nonparametric Bounds on Treatment Effects, The American Economic Review, 80 (2), 319-323.

[27] Manski, C.F. (1995). Identification Problems in the Social Sciences, Cambridge: Harvard University press.

[28] Manski, C.F. (2003). Partial Identification of Probability Distributions, Springer Series in Statistics: Springer-Verlag New York, Inc.

[29] Manski, C.F. (2007). Partial Identification of Counterfactual Choice Probabilities, International Economic Review, forthcoming. 
[30] Manski, C.F., and E. Tamer (2002). Inference on regressions with Interval Data on a Regressor or Outcome, Econometrica, 70, 519-547.

[31] Manski, C.F., and T.S. Thompson (1986). Operational Characteristics of Maximum Score Estimation, Journal of Econometrics, 32, 85-108.

[32] Manski, C.F., and T.S. Thompson (1989). Estimation of Best Predictors of Binary Response, Journal of Econometrics, 40, 97-123.

[33] Matheiss, T.H. (1973). An Algorithm for the Determination of Irrelevant Constraints and All Vertices in Systems of Linear Inequalities, Operations Research, 21 (1), 247-260.

[34] Matheiss, T.H., and David S. Rubin (1980). A Survey and Comparison of Methods for Finding All Vertices of Convex Polyhedral Sets, Mathematics of Operations Research, $5(2), 167-185$.

[35] Motzkin, T.S., H. Raiffa, G.L. Thompson, and R.M. Thrall (1953). The Double D Survey and Comparison of Methods for finding All Vertices of Convex Polyhedral Sets, Mathematics of Operations Research, 5 (2), 167-185.

[36] Padberg, M. (1999). Linear Optimization and Extensions, Springer-Verlag Berlin Heidelberg.

[37] Rosen, A.M. (2006). Confidence Sets for Partially Identified Parameters that Satisfy a Finite Number of Moment Inequalities, Working Paper CWP25/06, The Institute for Fiscal Studies and Department of Economics, University College London.

[38] Solodovnikov, A.S. (1977). Sistemy Linejnyh Neravenstv, Populyarnye lektcii po matematike, Vypusk 48: Izadatelstvo "Nauka", Moskva

[39] Vapnik, V.N. (2000). The Nature of Statistical Learning Theory, Statistics for Engineering and Information Science: Springer-Verlag New York, Inc. 